NIST Advanced Manufacturing Series 100-11

\title{
Proceedings of the 2017 Model-Based Enterprise (MBE) Summit
}

Thomas Hedberg, Jr.

Mark Carlisle

This publication is available free of charge from:

https://doi.org/10.6028/NIST.AMS.100-11

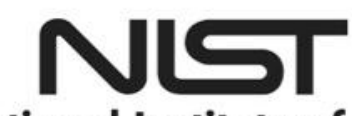

National Institute of Standards and Technology U.S. Department of Commerce 


\section{Proceedings of the 2017 Model-Based Enterprise (MBE) Summit}

Editors:

Thomas Hedberg, Jr.

Mark Carlisle

Systems Integration Division

Engineering Laboratory

This publication is available free of charge from:

https://doi.org/10.6028/NIST.AMS.100-11

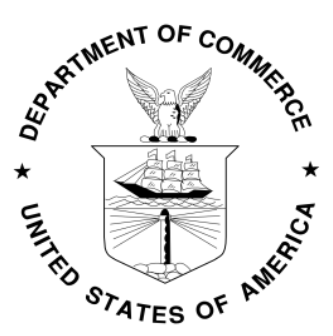

U.S. Department of Commerce Wilbur L. Ross, Jr., Secretary 


\section{DISCLAIMER}

This report was prepared as an account of work co-sponsored by National Institute of Standards and Technology (NIST). The views and opinions expressed herein do not necessarily state or reflect those of NIST. Certain commercial entities, equipment, or materials may be identified in this document to illustrate a point or concept adequately. Such identification is not intended to imply recommendation or endorsement by the NIST, nor is it intended to imply that the entities, materials, or equipment are necessarily the best available for the purpose.

National Institute of Standards and Technology Advanced Manufacturing Series 100-11 Natl. Inst. Stand. Technol. AMS 100-11, 44 pages (October 2017)

This publication is available free of charge from: https://doi.org/10.6028/NIST.AMS.100-11 


\section{Table of Contents}

1. Introduction...................................................................................................................................... 1

2. Keynote and Invited Speakers ................................................................................. 1

3. MBE Summit Trends........................................................................................................................... 3

3.1. Systems Engineering..........................................................................................................

3.2. Design.........................................................................................................................4

3.3. Manufacturing and Quality ...........................................................................................................4

3.4. Overall .........................................................................................................................................5

4. MBE Gaps and Challenges ..................................................................................................... 5

5. MBE Next Steps ................................................................................................................................... 6

6. Speaker Executive Summaries......................................................................................... 7 


\section{Introduction}

On April 3-6, 2017, the National Institute of Standards and Technology (NIST) hosted the Model-Based Enterprise (MBE) Summit to identify challenges, research, implementation issues, and lessons learned in manufacturing and quality assurance where a digital three-dimensional (3D) model of the product serves as the authoritative information source for all activities in the product's lifecycle. The MBE Summit was co-sponsored by NIST, the U.S. Department of Defense (DoD), and American Society of Mechanical Engineers (ASME). The 2017 theme was "Normalizing the Model-Based Enterprise." For the first time, there was an "MBE 101" session for attendees interested in learning about Model-Based Enterprise and the benefits MBE may bring to their companies.

The MBE Summit has developed into a key event for parties with an interest in advancing incorporation of digital 3D product models into activities throughout the product lifecycle. Held annually since 2009 at NIST, the Summit's evolution reflects the maturation path of MBE technologies. The first event concentrated primarily on improvements to DoD Technical Data Package (TDP) specifications. In subsequent years, topic areas increasingly expanded to address challenges not only in individual areas (design, manufacturing, inspection, etc.), but also in the mechanisms necessary to permit the uninterrupted flow of information across and beyond an organization's enterprise (e.g., supply chain). As organizations continue to work towards harnessing the benefits that an MBE environment can provide, the number and professional diversity of Summit participants have steadily grown each year. In the most recent years, these participants seem to share the sense that the largest impediments to implementation of MBE are no longer technological, but institutional. Although this indicates significant progress, many challenges remain. The Summit's organizers continue to seek the best means to help make MBE not only practical but prevalent.

The 2017 MBE Summit registration grew over 90\% from the 2016 MBE Summit. The 2017 MBE Summit program included one keynote, three invited speakers, four plenary presentations, an industry panel, and 40 technical presentations. In addition, at the end of the Summit, ASME held a workshop on forming MBE Standards. The DoD Digital Engineering Data and Modeling Working Group (DEDMWG) also met.

The MBE Summit began with an address from Dr. Howard H. Harary, the Director of the Engineering Laboratory (EL) at NIST. Dr. Harary spoke about a recent economic analysis ${ }^{1}$ released by NIST that found smart manufacturing advancements would save manufacturers $\$ 57.4$ billion per year. Smart manufacturing processes use digital information to optimize product, factory, and supply-chain operations. However, small enterprises face significant barriers to adoption of the needed technologies because they do not have the capital, like large enterprises and Original Equipment Manufacturers (OEMs), to support the types of investments required. Dr. Harary emphasized that enhancements in smart manufacturing are needed to enable technology and infrastructure adoption.

\section{Keynote and Invited Speakers}

The keynote speaker was Drura Parish, the CEO of MakeTime. Drura Parrish is a third-generation U.S. manufacturer and entrepreneur, who started MakeTime in 2013. Conceived as a two-sided online marketplace matching suppliers with excess capacity to manufacturers in need of parts, today MakeTime is a tech company dedicated to streamlining computer-numerical-control (CNC) machining production. MakeTime is developing technology that automates the procurement process, puts the supply chain online, and eliminates the request for quote (RFQ).

Mr. Parish spoke about the epistemology of manufacturing. In terms of the technological timeline, distributed manufacturing is relatively new. Enabled by the ubiquitous connections made possible through the cloud and the digitization of work, distributed manufacturing takes a decentralized approach to manufacturing that geographically localizes supply chains and federates them regionally. The result is a supply

\footnotetext{
${ }^{1}$ The Economic Impact of Technology Infrastructure for Smart Manufacturing, https://www.nist.gov/publications/economicimpact-technology-infrastructure-smart-manufacturing
} 
ecosystem that is, by nature, more stable and less precarious than its more traditional counterparts. Until recently, the risk mitigation offered by distributed manufacturing was its primary appeal. As exponential improvements in machine learning come online, however, big data is charting a course for a distributed manufacturing renaissance that belies all expectations. Given that distributed manufacturing just got here, Mr. Parish asked how can it already be in a renaissance.

Mr. Parish went on to explain that trying to make sense of and apply the troves of data we have generated and collected has been cumbersome due to a lack of proper tools. As powerful neural nets decrease in cost and increase in use, a lack of tools is fast-becoming a wealth of tools. Now, a distributed manufacturing base isn't just a path to more sustainable, local, and risk-free production; it's also a mine of relevant information and a playground for deep learning. We're at the edge of making discoveries - about processes, about prices, about parts and products themselves - we could have never made before. The next phase of manufacturing is an epistemological one. Data is remaking how, why, and what exists in real time and space.

In addition to the keynote and invited speakers, a plenary panel discussion was presented. The panelists were Ryan Kelly from MakeTime, Jack Baker from GoWesty - a MakeTime customer, and Rett Myers from Clark Machine Tool \& Die - a MakeTime supplier. The panel started with a discussion on the MakeTime model and how it enables both customers and suppliers to successfully achieve their goals. Topics discussed during the panel included model-based practices (i.e., paperless processes) and distributed manufacturing.

The summit also included three invited speakers, one each from academia, government, and industry. Dr. Leon McGinnis, Professor Emeritus from the Stewart School of Industrial and Systems Engineering at Georgia Institute of Technology, represented the academic sector, and spoke about the logistics domain. He said that while decisions about system design, planning, and control have major impacts on the cost and often the quality of the resulting product, logistics decision makers have no unified specification of their logistics systems, few computation decision support tools, and almost no end-to-end integration of decisions or analysis. Dr. McGinnis proposed that a major untapped opportunity exists to translate key learnings of MBE from the product domain to the logistics domain. He said that we can leverage the lessons learned from other domains using models (e.g., MBE, model-based systems engineering, computer-aided technologies (CAx)) to improve production systems decision making. The community can do this by integrating production-system knowledge into the system-design process.

Mr. Robert Gold, Director of the Engineering Enterprise Office of the Deputy Assistant Secretary of Defense for Systems Engineering of the United States Department of Defense (DoD), represented the governmental sector and spoke about the intersection of systems engineering and manufacturing. Mr. Gold highlighted the work in his office to support more systems thinking in manufacturing operations. Specifically, Mr. Gold's office is focused on integrating information by eliminating isolated data sources and connecting systems models to multiple knowledge repositories. Mr. Gold said the DoD is faced with several systems engineering challenges, one of which is the need for flexible designs that can adapt with innovation and are resilient to unknown missions and threats. This requires people to have integrated information available from across the lifecycle at the time design decisions are being made.

Lastly, Ms. Ambre Cauley, the Research and Development Portfolio Manager at Ingalls Shipbuilding, represented the industrial sector and presented how MBE alters the product lifecycle and industry with digital shipbuilding methods. Ingalls Shipbuilding is the largest employer in the state of Mississippi. Ms. Cauley presented some of the technologies that were developed and deployed in shipyards and the value those technologies brought to shipbuilding operations. In addition, Ms. Cauley highlighted the need to move towards more digital technologies, because the company has exhausted its ability to lower business costs and increase efficiencies in a paper-based environment. However, Ms. Cauley said business and people must continue to evolve and be open to technology changes. Areas in the shipyard being changed by digital technologies include data architectures, communication mechanisms, obsolescence management, information security, and U.S. Navy acceptance procedures. 
The remainder of this report will provide the reader with an overview of the output from the 2017 MBE Summit. Section 2 of this report highlights the major trends observed during the 2017 MBE Summit. Section 3 presents gaps and challenges in MBE that were identified by the MBE Summit attendees. Section 4 presents the MBE next steps that were discussed by the attendees. Lastly, the 2017 MBE Summit featured four technical tracks where speakers presented research and solutions related to MBE: (1) Systems Engineering, (2) Design, (3) Manufacturing, and (4) Quality. Executive summaries submitted by the speakers are included in Section 5.

\section{MBE Summit Trends}

An analysis of the speaker-submitted abstracts was conducted to investigate the topics and trends that were presented at the 2017 MBE Summit. Word clouds were generated to visualize the topics. The following sub-sections are the analysis results of the technical tracks and the overall Summit.

\subsection{Systems Engineering}

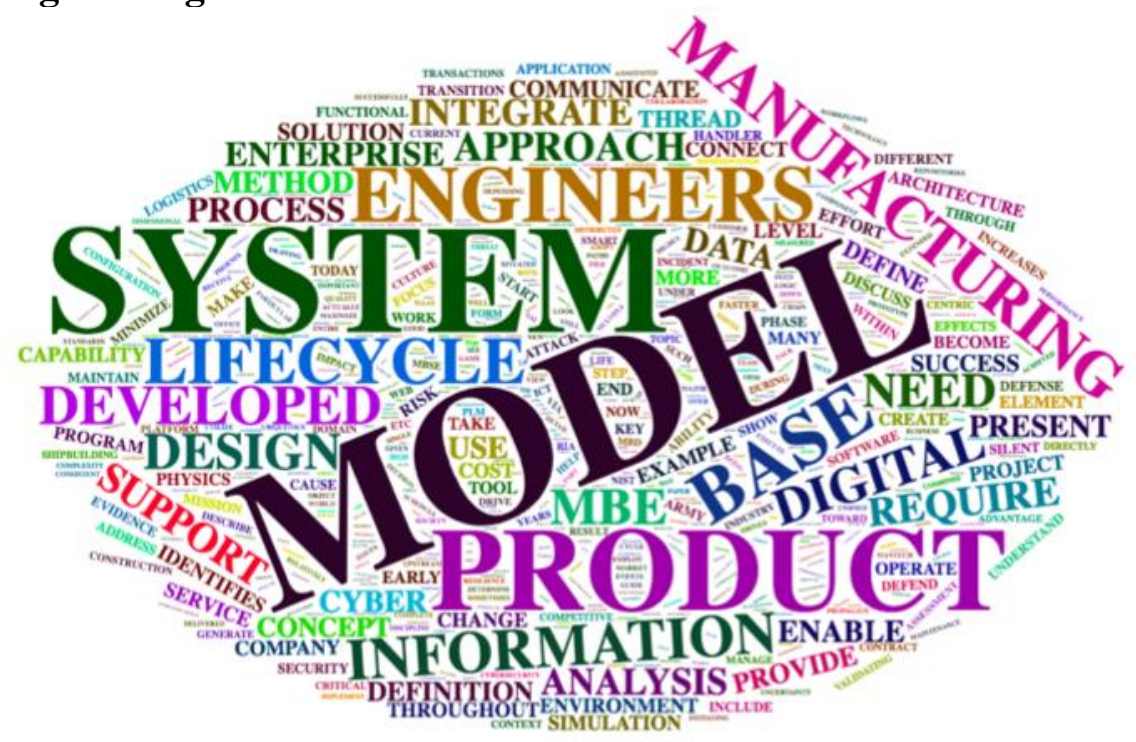

Figure 1. Word cloud of the topics presented in the Systems Engineering technical track.

Figure 1 presents the word cloud for the topics presented in the System Engineering technical track. 633 unique words were extracted from the abstracts of the presentations in the Systems Engineering technical track. MODEL, SYSTEM, and PRODUCT are the top three terms from the track. This should be no surprise because the Model-Based Systems Engineering (MBSE) is a popular trend currently in the Systems Engineering domain. 


\subsection{Design}

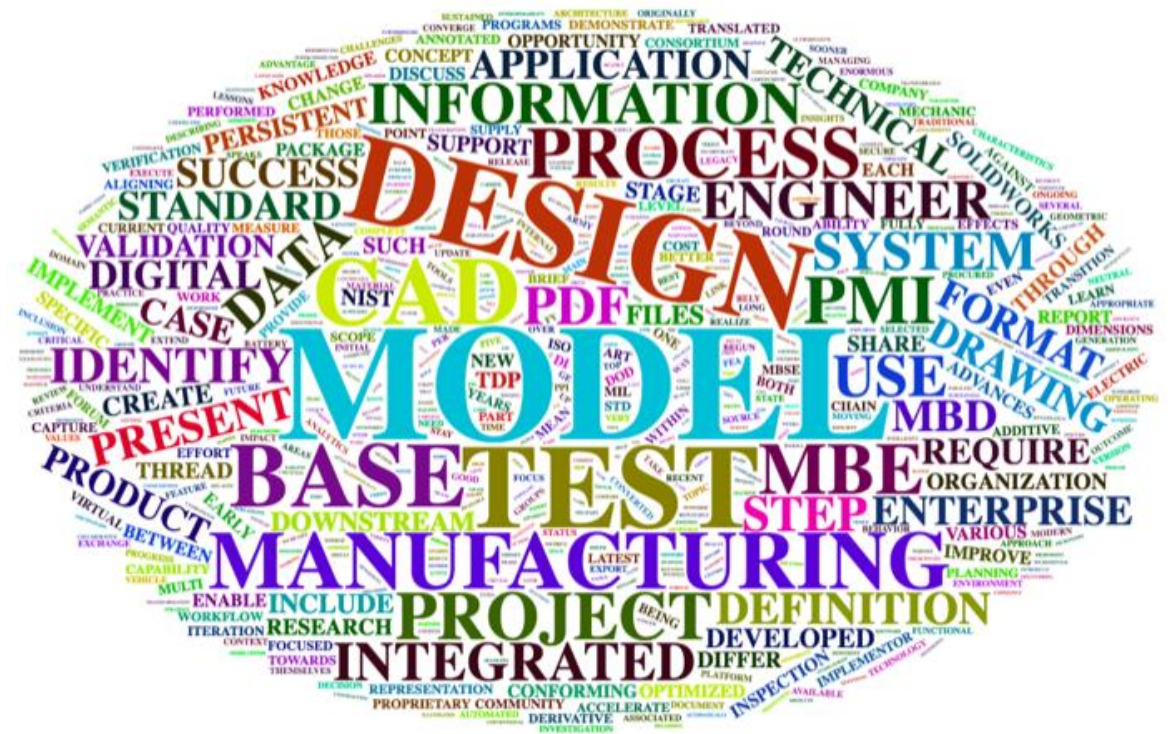

Figure 2. Word cloud of the topics presented in the Design technical track.

Figure 2 presents the word cloud for the topics presented in the Design technical track. 579 unique words were extracted from the abstracts of the presentations in the Design technical track. MODEL, DESIGN, and TEST are the top three terms from the track. MODEL is obvious because Model-Based Definition is a hot topic in the Design community. However, TEST was surprising.

\subsection{Manufacturing and Quality}

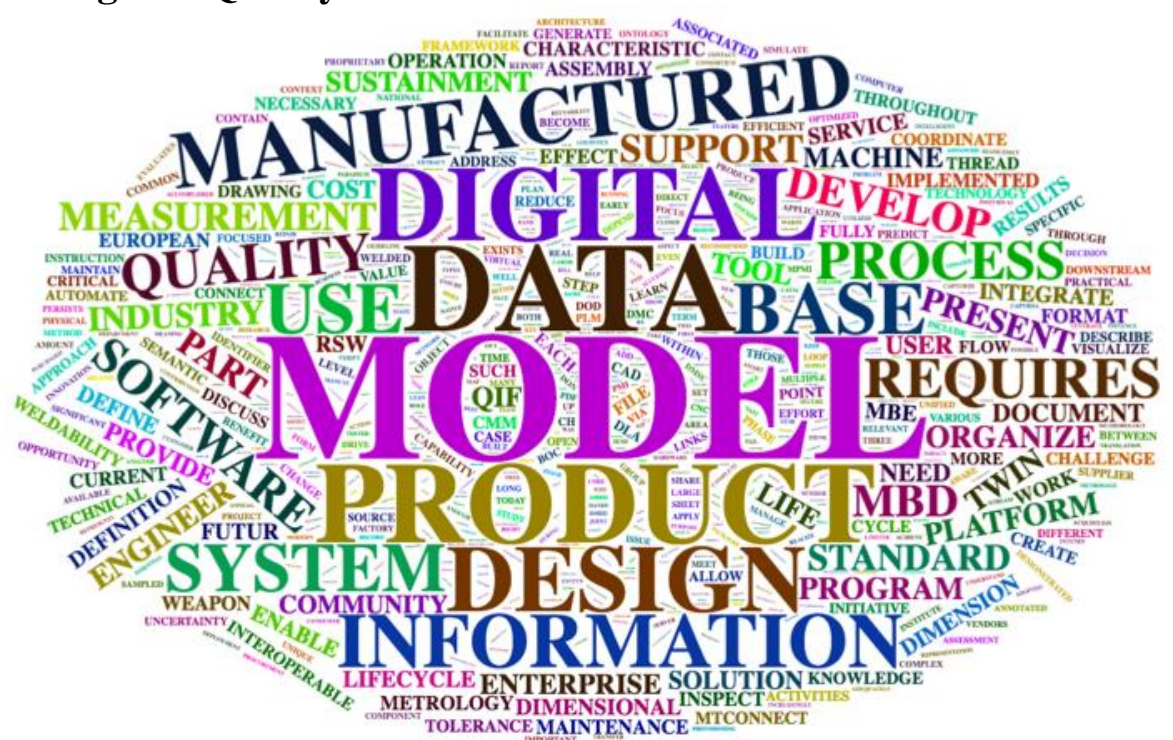

Figure 3. Word cloud of the topics presented in the Manufacturing and Quality technical track.

Figure 3 presents the word cloud for the topics presented in the Manufacturing and Quality technical track. 775 unique words were extracted from the abstracts of the presentations in the Manufacturing and Quality technical track. MODEL, DATA, and PRODUCT are the top three terms from the track. The results of the Manufacturing and Quality technical track align with the current trends (e.g., Model-Based Manufacturing, Data Analytics) observed within the Manufacturing domain. 


\subsection{Overall}

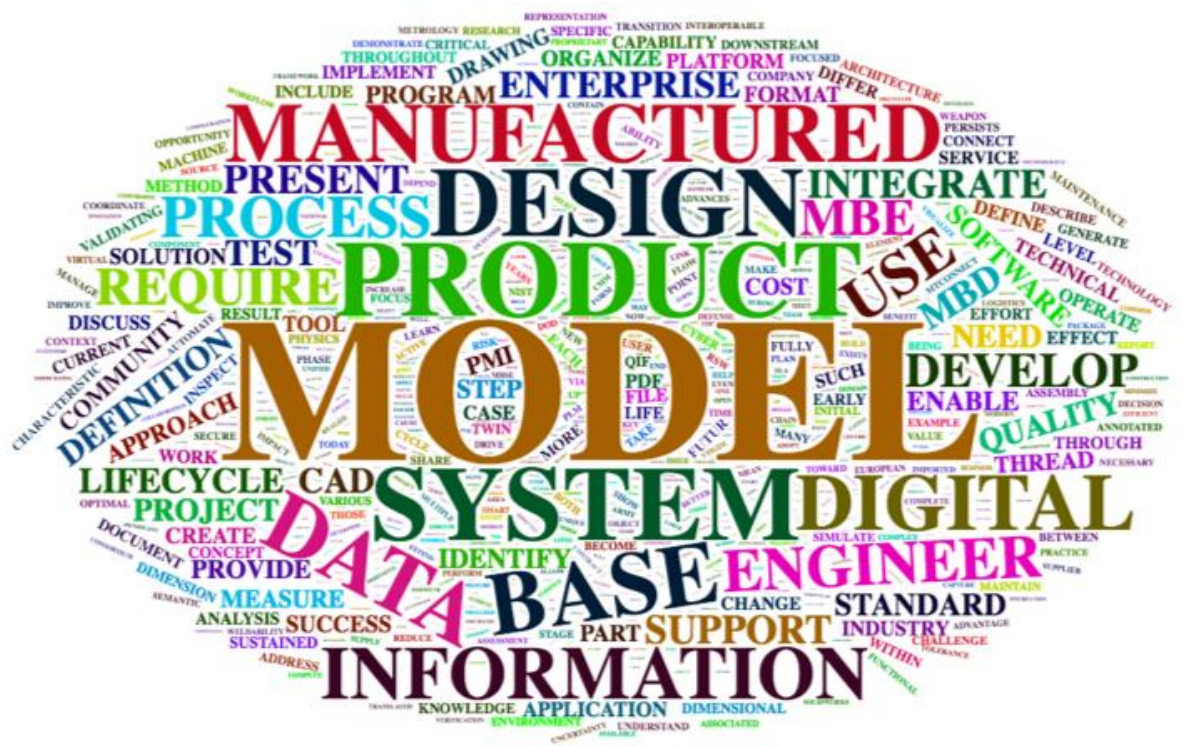

Figure 4. Word cloud of the topics presented in the entire 2017 MBE Summit.

Figure 4 presents the word cloud for the topics presented throughout the entire 2017 MBE Summit. 999 unique words were extracted from the abstracts of the presentations during the Summit. MODEL, PRODUCT, SYSTEM, DESIGN, DATA, MANUFACTURED, DIGITAL, INFORMATION, ENGINEER, and PROCESS are the top ten terms from the 2017 MBE Summit.

MODEL as the number-one term overall for the Summit is not surprising because each track and the Summit as whole are significantly interested in the models. In addition, the Summit is all about Model-Based Enterprise, which requires a model. However, something interesting was observed during the $2017 \mathrm{MBE}$ Summit. Technical Data Package (TDP) and Product and Manufacturing Information (PMI) were less prominent this year. For example, TDP ranks 254 out of 999. In years past, TDP would have ranked much higher. One reason for this shift in topics discussed during the Summit could be due to the maturing of the MBE concept and community. More solutions for MBE were presented this year. Whereas, gaps, challenges, and barriers in MBE dominated the discussion in previous MBE Summits. The community is beginning to discuss how to leverage MBE, which includes how data, information, and systems thinking can support industry adoption of MBE.

\section{MBE Gaps and Challenges}

While a shift toward solutions has been witnessed at the MBE Summit, gaps and challenges do remain. The Summit attendees were asked about the gaps and challenges in MBE that they face today. The following list is the output from the discussion about gaps and challenges that took place during the closing session of the 2017 MBE Summit.

- Getting cultures to change and be more open to automation. Recognizing that jobs roles may need to change to allow the automation

- The lifecycle encompasses the birth of an idea all the way to decommissioning of that idea

- Most of the discussion about MBE is starting in the middle of the lifecycle

$\circ$ Need to discuss more about the beginning of the lifecycle (e.g., stakeholder needs, trades studies) $\rightarrow$ Systems thinking

- Does the product lifecycle consist of only a single digital thread?

$\circ \quad$ How do we capture different contexts?

$\circ$ What about different viewpoints?

- How do we help the small-to-medium enterprises (SMEs) understand and/or gather requirements? 
- SMEs are struggling to figure out how to complete the model-based workflow

- Acceptable risk levels and definitions of the levels are needed

- Policy

- How do we write contracts well enough so we can enable MBE and affect positive change in culture?

- How do we influence the executives and business decision makers?

$\circ$ The people who have power-How do we legitimatize MBE to them?

- Cost benefits

$\circ$ We need more empirical data.

- How do we publicize the business benefit more widely? How can we show our upper management that there is an immediate benefit as well as a significant long-term benefit from MBE?

o How do we get our win-win-win solution? That is: how do we help the industry, our business, and our supply chain?

- Why not look at Building Information Modelling (BIM)?

- There are similarities and differences between manufacturing and the BIM world.

- Big difference: BIM users picks their suppliers at the very beginning. Manufacturing companies (e.g., OEMs) do not have as much control (as BIM users do) over picking suppliers earlier in the lifecycle.

- Legacy data and sustainment is a huge problem. The supply base is changing. The suppliers that were there 20 years ago, and were experts on those legacy systems, are no longer there today.

$\circ$ We are losing the industrial base and manufacturing support to ensure defense readiness and timely commercial availability repair parts.

- We need a MBE certification, like ISO 9001, for the supply chain

- Change management

$\circ$ This week, many solutions and studies that were presented are single iterations

- Why are we not talking more about changes?

- Industry needs better change-management processes with derivative datasets

- It is a big deal and a risk that needs to be managed.

- The Quality Information Framework (QIF) standard has "Qpids," which are persistent IDs. We need similar solutions in the other popular derivative datasets.

- Standards vs. solution differentiators

- Solution providers want to provide you tools that are better than the competition. But we want standards to help with interoperability. How do we overcome this problem?

- Need to reach consensus on terms such as "master" and "authorization"

\section{MBE Next Steps}

Given that the MBE Summit attendees identified a long list of gaps and challenges that remain in the MBE domain, the attendees were asked about the recommended next steps in MBE. The following list is the output from the discussion about next steps that took place during the closing session of the $2017 \mathrm{MBE}$ Summit.

- Trusted product models, data certification, and data traceability methods and solutions should be pursued. Also, industry needs trusted and tested solutions.

$\circ$ The quality role lives and dies by the Product and Manufacturing Information (PMI), but quality does not receive a guarantee that they can trust the models.

- For adoption to be successful, trust must be enabled.

- What is the definition of a good model today? It is the ability to reuse a design model in another computer-aided design (CAD) platform. Models need to be reusable in other downstream processes. 
- Manufacturing and other downstream consumption of model-based definition (MBD) models must be a higher priority than the drawing graphic sheet. Industry needs more downstream adoption of MBD.

○ There have been pilot projects; Industry needs realized commercial solutions

$\circ$ What are the requirements for PMI to help CAM? We know there is a big savings in coordinate-measure machines / systems (CMM/CMS). But how does Industry realize the same savings on the CAM side?

- How can we take the final product definition and help generate all the in-process models?

- Downstream processes need to demand MBDs from the upstream process. This would ensure upstream outputs align with downstream inputs.

- Need to define and standardize the use cases of MBD / MBE

- What does that workflow look like?

- Look at systems dynamics

- We need MBD recommended practices and practice standards (e.g., PMI to surface not an edge)

$\circ$ Training, retraining, knowledge management

\section{Speaker Executive Summaries}

The remaining pages of this report are executive summaries submitted by the presenters and/or speakers from the 2017 MBE Summit. The views and opinions expressed herein do not necessarily state or reflect those of NIST. Certain commercial entities, equipment, or materials may be identified in this document to illustrate a point or concept adequately. Such identification is not intended to imply recommendation or endorsement by the NIST, nor is it intended to imply that the entities, materials, or equipment are necessarily the best available for the purpose. 
Systems Engineering

\section{Digitalization of Systems Engineering - Examples and Benefits for the Enterprise}

\author{
Rob Beadling \\ DS Government Solutions \\ Waltham, MA, USA
}

\author{
Garrett Thurston \\ Dassault Systemes A\&D Industry Team \\ Waltham, MA, USA
}

\author{
Brian Chambers \\ DS Government Solutions \\ Waltham, MA, USA
}

\begin{abstract}
Across many industries, wherever products are comprised of automated systems of systems for improved performance, there is an increasing challenge that can only be solved through advanced system engineering processes. An example for defense is the Navy's surface combatant design challenge where future electric power loads will be driven by new, much more powerhungry weapons and sensors. The solution must be an optimized combination of power generation and energy storage systems. The Navy needs to architect, model, and simulate in accordance with real-world scenarios to ensure power is available for the ultimate combat effectiveness of future systems.
\end{abstract}

At present, most engineering organizations are still working in silos. Non-integrated file-based tools and file-based management systems are still the norm. The resulting disconnected tools and processes are very inefficient. Platform architectures are needed to accelerate engineering development and downstream processes. Platforms help businesses convert data that they have meticulously created and maintained in their legacy systems into corporate assets, paving the way for higher re-use and efficiency. Platforms digitalize the process along the value chain, providing real time access to all stake-holders in a transparent and auditable manner. Platforms also prepare businesses for the impending digital transformation.

A platform can be a framework to digitalize sustainable innovation. It unites engineers, suppliers, consumers and regulators, across the globe, and provides capabilities to model and simulate meaningful use case scenarios. With a platform, information flow and full traceability can be maintained across the system engineering pro- cess: from Requirements to Functional Architecture, to Logical Definition, to Physical Design. Related modeling and simulation I/O can also be associated with the correct version of the product/system as it develops for Validation. At the same time, the platform allows businesses to leverage their legacy data by indexing data in their existing systems to become more data-driven in their internal processes.

Analogies to the complex surface combatant design case above can be found in several places in Industry today. For example, all automotive OEMs are facing new challenges with Electric Car and Hybrid Electric Vehicle Development. Overall car architectures offer more possibilities: Rear or front Electric motor? Two Electric motors? Motor in wheel? Hybrid architectures also allow the possibility of integrating the Electric motor in the driveline. Core components like the batteries are undergoing massive evolution. Batteries are vulnerable to heat, aging, Charge/discharge cycles and other issues. Electrical motor performance is also altered by heat. Safety, fast charging, and longevity are big concerns.

To meet the challenges, BMW has developed and publicly documented their platform-based Dimensioning of Electric Drive System (DEDRIS). The aim of DEDRIS is to develop and fix the requirements to the electric drive system and its components during three iteration loops. Particularly when using modular components, a constant check between the requirements to the electric drive system and the achievable solution is necessary. This ensures that the choice of the components will lead to a consistent overall system design. The process is schematically shown in FIGURE 1 . 


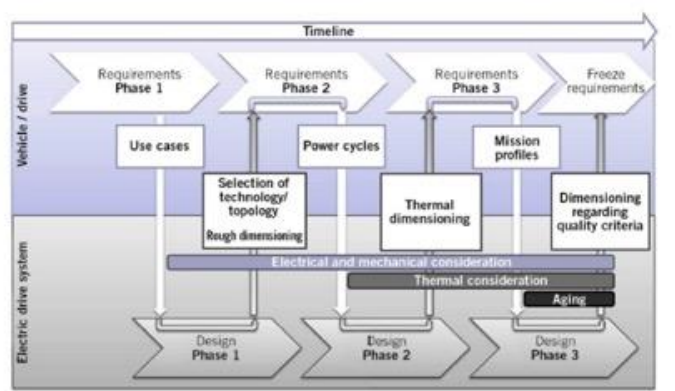

FIGURE 1 Process chart of the dimensioning of electric drive system (DEDRIS):

Meinert, Enger, Wiebkind, Diegelmann: The Plug-in Hybrid Technology of the New BMW X5 eDrive; MTZ

(Motorentechnische Zeitschrift), Issue 5/2015

BMW took advantage of multi-scale simulation capabilities. They also employed 1D simulations of dynamic behavior to model and simulate the performance of the integrated hybrid-electric drive train and chassis. The 1D simulation work was accelerated by the fact that it was fully integrated with their 3D modeling capability. The product of their efforts was the BMW X5 eDrive System.

Another high-end car company used a similar, platform-based, digitalized system engineering approach for hybrid-electric drive train development. They reported that what would normally have been a one-year project was reduced by 6 months. Their productivity also allowed them to build four prototypes instead of the three they had originally planned. Their efficiency enabled a $€ 2 \mathrm{M}$ savings for the project.

The complexity of engineering and manufacturing challenges that we face in business and our nation's defense are increasing, but so are the opportunities to advance our $R \& D$ capabilities to meet them. Digitalization and platform-based approaches can capture and manage all system requirements, allow experts to collaborate, and enable optimization through systematic physicsbased simulations. From the System of Systems view down the atomic-level, we can simulate performance to validate our conclusions before we commit them to hardware. A single source of truth for project data can be maintained throughout the process to ensure precise traceability of simulation models and results back to Requirements.

Our collective success in developing the highlyautomated systems of the future requires that we make development processes faster, more transactional and more integrated. Web-based and platform-based engineering streamline the accessibility and maintenance of consistent information. Technical solutions developed in online digital environments will also be able to feed Certification, Contract and Program execution as well as the digital manufacturing and lifecycle support of systems currently under development 


\title{
"Model Based (x) - Sparking a Systems Engineering leading practice for Innovative Pro- ject, Plant and Process Development"
}

\author{
Gerald Deren \\ Siemens Digital Factory \\ Phoenix AZ, USA
}

\begin{abstract}
As is the case with any competitive market, engineers are under constant pressure to finish their projects within tight schedule and at low cost so the company can get their products to market faster. It's a known fact that whoever does it will have an added advantage over their competitors driving many to seek a Model Based approach towards solving that challenge.
\end{abstract}

Model Based is not a new concept and actually has been become a core competency many companies across many industries over $20+$ years. In the early 80's, Model based was referred to as the "Master Model" concept or part/product centric design. Numerous companies' product development domains have embraced this approach with great success and have been able to execute to enjoy a competitive advantage.

Having experienced that success, and seeing their competition do so, many companies are looking towards this approach to better work within their environments.
A key learning is that "Model Based" has started to take on different forms of focus, depending on what part of the life cycle you are actually working in.

$$
\begin{aligned}
& \text { MBSE = Model Based Systems Engi- } \\
& \text { neering } \\
& \text { MBE = Enterprise, Engineering, Envi- } \\
& \text { ronment... } \\
& \text { MBD = Design, Definition, Drafting... } \\
& M B M=\text { Manufacturing... } \\
& M B Q=\text { Quality }
\end{aligned}
$$

But the key to success is the way they start their journey in their systems engineering practice. The focus of this topic is to discuss a PLM based $\mathrm{MB}(\mathrm{x})$ approach and discusses some of the gains certain customers have seen. $\mathrm{MB}(\mathrm{x})$ is NOT a single answer and does not define a correct way for all to adopt it but it does make visible the need for companies to enable a discipline with respect to communication, collaboration, and measurement throughout the entire product development lifecycle. 


\section{Defining the Minimum Information Model for a Model-based Definition}

\author{
Nathan W. Hartman, Ed.D. \\ Purdue University \\ West Lafayette, IN, USA \\ Jesse Zahner \\ Purdue University \\ West Lafayette, IN, USA
}

\author{
Alexander Miller \\ Purdue University \\ West Lafayette, IN, USA
}

In a model-based enterprise (MBE) the "model" serves as a container, not only of shape definition (i.e., geometry, PMI, annotations, etc.), but also of behavioral (i.e., materials, functional logic, genealogy, etc.) and contextual (i.e., supply chain, in-use, assembly, etc.) data as well - a model-based definition (MBD). As industry transitions to the use of annotated 3D models in lieu of 2D drawings as a communications mechanism, there is ongoing debate about which information elements to include in the model.

The minimum information model (MIM) is not a blanket that fits over every industry sector and job role. An important differentiation stated in this study was that of the difference between the "minimum" information model and the "common" information model. The common information model (CIM) is all information related to the product definition at any stage in the lifecycle. It assumes to represent the model-based information for the lifecycle, where any information that is used or necessary would be included. The minimum information model (MIM) is the minimum information needed to conduct activities in a specific workflow. It does not persist beyond the workflow in which it is currently used. A designer's minimum information model is different from a machinist's minimum information model, which would again be different for a supplier manager. Understanding the characteristics of the data needed during these lifecycles is important to both adequate compliance, information fidelity, and to minimizing costs. This research study aimed at identifying the necessary information elements in a model as the model progresses through the lifecycle.

The first stage of the study used a broad survey to identify elements of the minimum information model, and assess industry adoption of MBD techniques. Participants responded regarding the information that they needed to complete the tasks assigned to them within their respective workflow, as well as their typical medium for information exchange, and the capabilities of software to carry the information they needed. This provided insight into both the requirements of the minimum information model, and the user's perception of model-based definition tools. A minimum information model did not result from this stage, but it did provide a more complete understanding of the problem. This understanding was a foundation for a follow-on research effort through a Delphi study.

The second stage of the MIM study used the Delphi method to reach expert consensus. The Delphi Round 1 survey asked questions about workflows, roles within the company, and elements necessary for a specific workflow. It also included whether the respondent used models, drawings, or a combination of both. All questions for Delphi Round 1 aimed at identifying information needed for a specific workflow, but also identified information deemed as unnecessary or lost throughout the product's lifecycle. Twenty-two information elements resulted from Delphi Round 1. Delphi Round 2 survey asked participants to rate the twenty-two elements on a scale from one (not important) to seven (very important). Participants responded twice: once in regards to their workflow and once in regards to the lifecycle (ignoring workflows). Interviews of ten industry practitioners during Delphi Round 2 provided context to the survey responses. Round 2 of the Delphi resulted in ten of the elements being either redundant or completely unnecessary, so they were eliminated. Delphi Round 3 is currently underway to determine the importance of the unique information elements from Round 2. 


\title{
Good Testing is Hard, Bad Testing is Easy
}

\author{
Robert R. Lipman \\ National Institute of Standards and Technology \\ Gaithersburg, MD, USA
}

Software and standards are key enablers of the Model-Based Enterprise. Design software is used to generate 3D model-based designs (MBD) from product requirements. ASME and ISO standards specify how product and manufacturing information (PMI) is created in a CAD software. Standards also specify how information is stored and exchanged through derivative file formats.

The NIST MBE PMI Validation and Conformance Testing Project created a test system to measure conformance of CAD software to ASME standards for geometric dimensioning and tolerancing. The test system has five main components: (1) test case definition and expert review, (2) test CAD model creation based on the test case definition, (3) verification of the CAD models against the test case definitions, (4) generation of derivative STEP, JT, and 3D PDF files by the Implementor Forums and, (5) validation of the derivative files against the CAD models and test case definitions.

There are many different types and characteristics of testing. Conformance and interoperability testing tests the transfer of information between two systems. Syntax, structure, and semantic testing tests information in derivative files. Representation and presentation testing tests semantic and graphic PMI. Good testing also requires a well-defined scope, testing methodology, test cases, testing criteria, testing tools, and results reporting.

Did the NIST testing project do good testing (hard) or bad testing (easy)? The nine test cases that were modeled in four CAD systems contain a wide variety of geometric and dimensional tolerances (GD\&T), however, the GD\&T was not intended to represent best practice. In that respect, it tested CAD software very well for modeling PMI but might not be representative of an end-user's use of GD\&T. A lot of verification and validation testing results were collected, in a semi-automated method, regarding the modeling of semantic and graphic PMI. Detailed results were shared with the CAD system developers so that issues could be addressed. However, for the public, the raw results were distilled into percentages of success for modeling PMI in several graphic and semantic categories. The methodology did not weight the raw results for the importance of a type of error. Graphic PMI errors that can be correctly interpreted by a human were given equal weight to semantic PMI errors that would affect the downstream consumption of the information by manufacturing and inspection processes. The distribution of the types of GD\&T in a test case can also skew the results. Better methodologies need to be developed to identify the importance of PMI verification and validation errors.

Overall the NIST testing project has been a great success in terms of identifying issues with how PMI is modeled in CAD software and exported to derivative files which has resulted in better CAD software for the end-user. All the project's test cases, CAD models, STEP files, and results are available at https://go.usa.gov/mGVm.

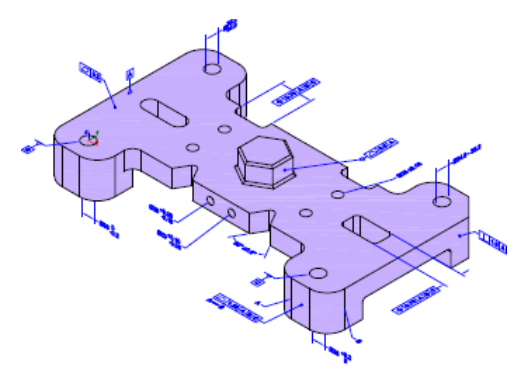




\section{Comply with the NIST PMI Test Requirements using SOLIDWORKS}

\author{
Oboe Wu \\ DS SOLIDWORKS \\ Waltham, MA, USA
}

\author{
Christopher Pagliarini \\ DS SOLIDWORKS \\ Waltham, MA, USA
}

\begin{abstract}
The national institute of standards and technology (NIST) initiated the MBE PMI Validation and Conformance Testing Project in 2012 to help measure and improve the product and manufacturing information (PMI) quality by four CAD systems. SOLIDWORKS was selected in the project since the inception. From 2012 to 2017, SOLIDWORKS has made substantial progress to comply with the PMI test requirements and support practical manufacturing needs.
\end{abstract}

The enhancements can be categorized into two areas, PMI definition and PMI organization. For PMI definition, the primary focus has been on the representational PMI to drive the downstream manufacturing automations. For example, SOLIDWORKS Model-Based Definition (MBD) 2016 added the capability to automatically create and display the corresponding coordinate system upon selecting a feature control frame. This enhancement not only facilitates the human comprehension of the geometric dimensioning and tolerancing (GD\&T), but also opens API calls for manufacturing software applications to reuse the coordinate systems.

Besides the representational PMI definition, special effort has been committed to improving the end user experiences to ease the transition from $2 \mathrm{D}$ drawings to MBD. For example, the selection of features has been much easier in SOLIDWORKS MBD 2017 than in SOLIDWORKS 2012. Rather than having to select the inner face of a hole, users can simply pick a hole edge, similar to the 2D drawing convention. SOLIDWORKS then intelligently presents users with the most probable feature definition options based on the selected edge.

Next, PMI organization is the key to making the PMI consumable and actionable. The NIST test cases all defined specific views to organize the models and annotations. SOLIDWORKS MBD 2015 introduced 3D Views to better comply with these NIST requirements. 3D Views allow users to create visual bookmarks of specific orientations, zooming factors, display states, annotation views, model styles and configurations. This comprehensive capturing of a model and PMI can help users build a storyline of the design specifications for the downstream data consumers.

Additional PMI improvements beyond the current NIST test scope are also added to the ongoing releases, such as the 3D PMI comparison between revisions, STEP242 export per the ISO standard 10303-242:2014 and the viewing of CAD models and PMI from other CAD platforms in eDrawings $\underline{2017}$ per the military standard 31000A:2013.

With the solid enhancements from SOLIDWORKS 2012 to 2017, SOLIDWORKS users can meet the NIST PMI test requirements with much better functionalities and greater confidence. From the industry's perspective, a larger number of high quality models with PMI will be created and distributed to assist the overall advancement of model-based enterprise (MBE) initiatives. 


\title{
Evolve or Dissolve - Effective MBD/MBE Strategy and Benefits
}

\author{
Jennifer Herron
}

Action Engineering

Lakewood, CO, USA

Discovering your organization's primary motivation and pain points are essential to effectively implement an MBD/MBE strategy. Action Engineering's CEO shared three top areas for companies to focus in order to be successful in implementing Model-Based Definition (MBD) inside a Model-Based Enterprise (MBE).

Smart manufacturing requires a digital enterprise. A digital enterprise requires digital data. Digital data for production definition is call Model-Based Definition (MBD). When smart engineering is digitally associated with smart manufacturing, then the Return on Investment (ROI) can be fully achieved. Using the QIF format created after MBD is defined on a product to create QIF MBD, QIF Plans and to execute product measurements is an example of generating savings through automation during the downstream product lifecycle.

Today MBD has four parts, 3D geometry, Annotations*, Attributes* and Presentation* (* As defined in the ASME Y14 series). MBD is different from drawings because the MBD author creates unambiguous definition of the product where there is no room left for human interpretation. Thus, per trends we see from Lifecycle Insights, using MBD- based product definition, we may begin to reduce scrap rates of $40 \%$, and reduce the amount of change orders by $9 \%$.

If you are an organization ready to begin your MBD/MBE journey, first acknowledge your pain points, and measure them. Determine and define if an $\mathrm{MBD} / \mathrm{MBE}$ strategy will reduce that pain. Evaluate your organization by examining Design, Manufacturing, Quality, Procurement, and Data Management processes at a detailed level. At this point, business value can be determined and an effective MBD/MBE roadmap and strategy can be derived and executed. Once your organization has evolved from a low MBD/MBE capability to a Best-In-Class capability, then your continuous improvement processes may be able to eliminate human consumption elements in place in MBD today, such as Presentation.

However, it is easy to get overwhelmed. Action Engineering recommends organizations focus on these top three strategies: Robustly evaluate software tools and plan for regular maintenance of those tools, Define MBD and execute consistently, and Derive a robust CAD source, derivative and interoperability strategy. 


\section{Automatic Generation of Optimized CMM Program from MBD on the DMDII Digital Manufacturing Commons and Enabled by QIF}

\author{
Daniel Campbell \\ Metrosage \\ Sugar Land, TX, USA \\ Victor Mikushin \\ Capvidia \\ Leuven, Belgium \\ John Schmelzle \\ US Naval Air Warfare Center (NAVAIR) \\ Lakehurst, NJ, USA
}

\author{
James Barkley \\ DMDII \\ Chicago, IL, USA
}

\author{
Dr. Ed Morse \\ University of North Carolina Charlotte \\ Charlotte, NC, USA \\ Murray Desnoyer \\ Origin International Inc. \\ Toronto, ON, Canada
}

As GD\&T requirements are increasingly incorporated into the Model Based Definition (MBD) in the form of semantic Product and Manufacturing Information (PMI), direct and automated CMM program generation becomes possible, significantly reducing errors and labor costs. Moreover, in recent years the quality community has become increasingly aware of the implications of measurement uncertainty and its role in pass/fail assessments risks. Assessment of task-specific measurement uncertainties in coordinate metrology via simulation is now a reality, and provides a basis for optimizing measurement strategies to reduce measurement uncertainties, and for the reporting of the resultant task-specific uncertainty value for each GD\&T assessment. The ability to generate optimized CMM programs directly from the MBD represents a dramatic innovation in dimensional quality control.

To ensure access to this cutting-edge technology by large organizations and SMEs alike, Metrosage,
Capvidia, UNCC, and NAVAIR have teamed up with the Digital Manufacturing and Design Innovation Institute (DMDII) to use their Digital Manufacturing Commons (DMC) as a platform. The DMC is an open-source, open architecture communication platform which enables plug-andplay functionality across the entire digital thread. It facilitates systems engineering across the manufacturing enterprise by allowing for the aggregation, storage, and analysis of product data by modular processes.

Software packages from Capvidia, Metrosage, and Origin International will be linked together on the DMC by use of the Quality Information Framework (QIF) data format. This ANSI standard allows for the propagation of semantic MBD and Measurement data.

Deployment of this automated tool on the DMC will enable an online community of users from organization of all types and sizes to benefit from the latest in advanced MBD technology. 


\section{CH53K Design Models; Can They Support System Sustainment Requirements?}

\author{
Thomas K. Parks \\ Senior Fellow, LMI \\ McLean, VA, USA
}

\author{
Dick Tiano \\ Senior Program Manager, ATI \\ Summerville, SC, USA
}

The CH-53K King Stallion helicopter program is currently in the Engineering and Manufacturing Development Phase of the acquisition cycle. The program intends to design, develop, and maintain the platform using three-dimensional (3D) models documented in CATIA computer-aided design (CAD) software. ${ }^{2}$ The program's planned approach for delivering 3D technical data as CAD models/files to support system sustainment presents a challenge for the Defense Logistics Agency (DLA) since their current provisioning and procurement processes are built to accommodate and use twodimensional (2D) technical data, not 3D models.

CH-53K 3D model issues relative to supporting the sustainment process are not unique. A number of other developing Department of Defense (DoD) weapon system programs plan to use $3 \mathrm{D}$ models as part of a model-based enterprise (MBE) approach throughout the system's life cycle. Unfortunately, guidance regarding 3D data completeness and format requirements is lacking. Additionally, the system designers who develop the native CAD files, which should be the basis for all follow-on manufacturing and sustainment activities, often do not include (or even consider) the requirements for these activities in the baseline 3D models. Exacerbating this basic issue is the need to transfer model data to sustainment organizations in a format they can use (i.e., neutral file format) instead of the CATIA software format. Addressing the issues of adequately documenting and transferring 3D model data is extremely important for DLA and the DoD because many new weapon systems will soon be engaging DLA for assistance and sustainment support.

DLA and the $\mathrm{CH}-53 \mathrm{~K}$ program have identified a solution for the 3D technical data challenge, that may become the benchmark for addressing similar challenges with other DoD weapon system acquisition programs. Specifically, they found that the $3 D$ PDF plus STEP file format is the best solution for providing CH53K 3D technical data to support sustainment. ${ }^{3}$ But this solution will only work if the base models are adequately documented and annotated.

The proposed solution, if adopted by DoD, will have a direct effect on system designers and the way MBE is implemented across the Military Services' weapon system programs. To assist DLA, PMA261, and other Program Offices in calculating the basic and expected annual costs of implementing a 3D PDF solution, we compiled all of the requirements and the associated labor hours and costs into a cost analysis tool.
2 CATIA software is Dassault Systems' proprietary 3D interactive application and product development platform for creating system design models.
3 "STEP" is the informal term for "Standard for the Exchange of Product model data." 


\title{
MBD Implementation Dos and DONTs
}

\author{
Oboe Wu \\ DS SOLIDWORKS \\ Waltham, MA, USA
}

Model-based definition (MBD) practices have gained substantial traction in the past ten years from 2007 to 2017 as of this writing. For example, a series of SOLIDWORKS customer base surveys suggested that the percentage of manufacturers who define or plan to define annotations directly to 3D models rather than 2D drawings has increased from 1.5 percent in 2009 , to 16 percent in 2013 , then to 20 percent in 2015. Figure 1 showed the Geoffrey Moore technology adoption curve, onto which the above percentages are plotted.

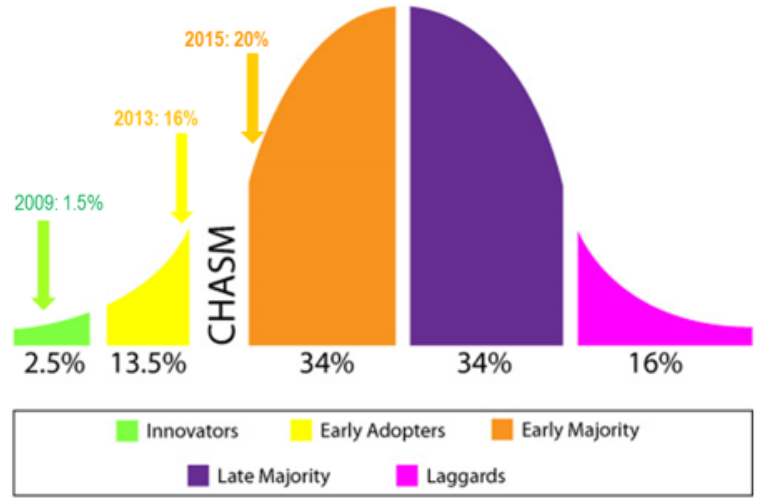

Figure 1. Do you define or plan to define annotations to your 3D models? (Survey sample sizes: 700 in 2009, 847 in 2013 and 524 in 2015).

In this growing trend, the common question about MBD is transitioning from "why implement" to "how to implement". However, the implementation scope is comprehensive and well beyond software or hardware tools. A successful implementation requires significant shifts across the extended enterprises including both internal teams and external suppliers. A wise approach for interested manufacturers is first to understand the lessons learned by peers, which can help avoid repeating the same mis- takes and follow recommended practices. Therefore, Table 1 collected practical implementation experiences from dozens of MBD pioneers around the globe as DOs and DONTs from the people, process and product aspects. These three high-level aspects are explained as follows:

People: human mindset and organizational structures.

Process: methodologies, procedures, and tools.

Product: product design, manufacturing and maintenance.

Table 1. MBD Implementation DOs and DONTs

\begin{tabular}{|l|l|l|}
\hline $\begin{array}{l}\text { Implementa- } \\
\text { tion aspects }\end{array}$ & DOs & DONTs \\
\hline People & $\begin{array}{l}\text { Secure the } \\
\text { leadership } \\
\text { support }\end{array}$ & $\begin{array}{l}\text { Don't take too } \\
\text { big a bite at } \\
\text { first }\end{array}$ \\
\hline Process & $\begin{array}{l}\text { Automate } \\
\text { workflows }\end{array}$ & $\begin{array}{l}\text { Don't exclude } \\
\text { printouts }\end{array}$ \\
\hline Product & $\begin{array}{l}\text { Organize and } \\
\text { present anno- } \\
\text { tations clearly }\end{array}$ & $\begin{array}{l}\text { Don't skip } \\
\text { calling out } \\
\text { critical annota- } \\
\text { tions }\end{array}$ \\
\hline
\end{tabular}

Both near-term and long-term recommendations are discussed, but the focus is on relevant and practical solutions as of 2017 because the immediate priority is to ease the transition from the $2 \mathrm{D}$ drawing processes to model-based processes, rather than ideal workflows in ten years. 


\section{Model-based Product Manufacturing Information (MPMI)}

\author{
Tom Rando \\ Electric Boat Corp. \\ Groton, CT, USA
}

\author{
Heidi Preston \\ Electric Boat Corp. \\ Groton, CT, USA
}

\begin{abstract}
Previously, the engineering drawing was the document of record that captured the normative directions for building, inspecting and testing. The drawing-based process had the advantage that its information was authoritative and complete. It had the drawback that it was too coarse grained to support a lean work instruction. An engineering drawing contains appreciably more dimensions than apply to a particular operation. The smallest addressable unit of dimension data is the drawing sheet, but sheets, too, contain many more dimensions than needed for an operation.
\end{abstract}

The 3D model contains enough information to define fully the form and fit of ship components; paradoxically, it does not contain enough information to build or inspect systematically. From among the infinitude of dimensions latent in the 3D model, there are a handful of dimensions needed to fabricate, assemble, install or inspect each component piece of the submarine. The MBE challenge is to determine automatically which dimensions are those critical dimensions. Each such critical dimension is an entity defined by a set of data, and this entity can be called an MPMI object.

A common misconception is that moving to $3 \mathrm{D}$ is what makes an IPDE 'model-based.' In fact, what constitutes 'model-based' is when there is a digital model in which the important components of an enterprise or product model are objects with identifiers (names) that are available to users and to software applications. An MPMI object must have a persistent database-friendly identifier. The paper describes an approach to select, author and manage MPMI objects that support lean 3D work instructions. 


\section{Don't Do Digital Design Wrong: Use of 3D Technical Data Doesn't End at Manufacturing}

\author{
Thomas K. Parks \\ Senior Fellow, LMI \\ McLean, VA, USA
}

\author{
Dick Tiano \\ Senior Program Manager, ATI \\ Summerville, SC, USA
}

Today's MBE discussion focuses on the early phases of the digital thread, design and manufacturing, and rightly so. It is imperative that we efficiently and effectively develop and engineer weapon system designs that meet their intended purpose/requirements and that can be manufactured economically and rapidly. But, there is another, critical, often overlooked, aspect of getting the design right. The design as documented in the model should be the bedrock for supporting and maintaining a weapon system throughout its operational life.

Between $65 \%$ and $80 \%$ of all weapon system life cycle costs are incurred during operations and sustainment, a period that spans $80 \%$ to $95 \%$ of the system's life. Not thinking ahead and failing to build the model for sustainment can cripple a program for decades, driving up costs and driving down readiness. 3D design done right is an enabler of smart sustainment.

So, how are operations and sustainment impacted by the design models developed during the early phases of the digital thread? All of the cataloging and provisioning documentation that defines each part should be based on the digital model/design. Every part used for repair and planned maintenance should be produced using the digital model/design. Every technical manual and work instruction for carrying out those repair and maintenance actions should be based on the digital model/design. Every system design change and every corresponding part that needs to be built, stocked, and maintained, should be based on the digital model/design.

System engineers and designers creating the digital models need to think about what happens with their product/model downstream in the life cycle and ensure that it can meet the associated requirements.

\footnotetext{
4 "STEP" is the informal term for "Standard for the Exchange of Product model data."
}

\begin{abstract}
Maintenance - 3D models can and should flow seamlessly into bills of material, integrated parts breakdown, maintenance instructions, and integrated electronic technical manuals.
\end{abstract}

Configuration management - as changes are made to the system, models should be updated and flow seamlessly throughout maintenance and supply. The days of record of changes, for twice annual updates should be a thing of the past.

Procurement -if a part is required for maintenance and it is not stocked, it must be purchased from a manufacturer. In this instance, a well-documented model, annotated with essential metadata is an absolute prerequisite for purchasing the part. But, there's more to it than just documentation and annotation. The model must be readable and useable by all the prospective manufacturers to meet government 'fairness requirements' for contracting.

The Defense Logistics Agency (DLA) in conjunction with several Service engineering support activities (ESAs) has identified a solution for the 3D technical data challenge, that may become the benchmark for addressing similar challenges with other DoD weapon system acquisition programs. Specifically, they found that the 3D PDF plus STEP file format is the best solution for providing 3D technical data to support sustainment. However, this solution will only work if the base models are adequately documented and annotated during the design phase. 


\section{CAD Reusability and the Role of Modeling Information in the MBE Context}

\author{
Jorge D. Camba \\ University of Houston \\ Houston, TX, USA
}

\author{
Pedro Company \\ Universidad Jaume I \\ Castellón, Spain
}

\author{
Manuel Contero \\ Universidad Politécnica de Valencia \\ Valencia, Spain
}

\begin{abstract}
Modern approaches to product development rely on digital 3D models as the primary data source for all activities throughout the product life cycle. In this context, the quality of the CAD model, which is inherently rich in information, and the efficient flow of information from design to manufacturing and other support processes are crucial.
\end{abstract}

Many efforts in the area of MBE have focused on optimizing the flow of information running through the product's lifecycle. But current mechanisms can also facilitate the flow of information across single activities by integrating unstructured information in a manner that facilitates reusability and communication, and centralizes design knowledge, thus creating even richer models.

Our vision is that improving digital product representation and data quality and its connection to design knowledge can lead to more efficient product development processes. By improving the quality of the digital data source at the early stages of the product lifecycle, the need for revisions, redesigns, and back and forth communication can be drastically reduced, which maximizes the reusability of the digital product and improves subsequent downstream processes. The execution of this vision is based on three primary ideas: (1) ensure digital product models and modeling processes achieve the highest possible quality at all linguistic levels while they are being created, (2) analyze, correct, and repair defects (particularly at higher semantic levels) to guarantee the reliability and robustness of master digital models, and (3) enrich models with design information and mechanisms to centralize knowledge and facilitate design communication.

Just as the quality of a software system depends on the correctness and efficiency of its code, the quality of products depends on the quality of their design processes, which then depend on the quality of their data. In this regard, there are currently no formal criteria to reliably implement and assess the correctness, validity, and efficiency of a digital product model and its associated data. Current strategies rely on qualitative guidelines based on user experience or existing practices, which may contain biases and dependencies. This lack of understanding has evolved into inefficient digital models built through trial and error and excessive use of brute force. Designers have become adept at building reasonably reliable models out of unreliable parts, which usually leads to maintainability and reusability problems. In fact, just understanding the internal structure of an existing model can be extremely difficult even for its original creator after a certain amount of time. These models can easily result in significant losses in terms of time and production costs. Finally, as digital models gradually become more complex and information-rich, the availability and access to design knowledge becomes even more relevant. Therefore, our understanding of digital design and manufacturing would not be complete without developing these fundamental concepts. 


\title{
Activities on Product Service Platforms Interoperability in Europe
}

\author{
Dr.-Ing. Dipl.-Kfm. Christoph Runde \\ Intelligent Manufacturing Systems (IMS) \\ Brussels, European Union
}

The increasing economic importance of pre- production and post-production value creation (in relation to manufacturing itself) indicates the need for product-service- systems (PSS) and for platforms to design PSS. The creation of such PSS design platforms is very challenging, because PSS development needs to take into account all PSS relevant stakeholders (e.g. product manufacturer, customer, sensor and part suppliers, production and process engineers) and all PSS relevant data sources (e.g. design data, manufacturing data, MES data, production process data, usage date, ...). Establishing PSS thus means a significant integration task. Integration in turn postulates interoperability.

Interoperability in PSS development is necessary; however, there are interoperability hurdles, which can be segmented into three pillars: syntactic, semantic and organizational challenges. Syntactic interoperability hurdles include, how different sources of product usage information (PUI, such as PEIDs, operation logfiles, maintenance logfiles, social media networks, helpdesks, ERP systems) can be integrated with target IT systems in PSS development (e.g. CAx, simulation, forecasting, data analytics). Semantic integration issues address how information and knowledge from different lifecycle phases, processes and domains can be integrated into the PSS development processes and systems. This is required to address general PSS and sector-specific requirements. Organizational interoperability challenges are caused by multiple stakeholders' integration needs, including 3rd parties such as social media networks, actors from different processes and knowledge domains (e.g. PSS design, operation, maintenance, customers and end-users from both $\mathrm{B} 2 \mathrm{~B}$ and consumer contexts).
Within the European Union's initiative in the area of the Factories of the Future, a number of running European projects (Connected Factories, Diversity, Falcon, ICP4Life, Manutelligence, ProRegio and Psymbiosys) deals with the development of integrated product service systems. The projects address interoperability aspects and contribute to standards in this field (ODF, O- LM, O-MI, OSLC, RAMI4.0, OWL, STEP, KbeML,...). The above mentioned projects met in Brussels on November 21, 2016 to exchange their views on PSS platform interoperability. Key questions discussed were, if existing frameworks as RAMI4.0 could serve as an integration platform, which extensions are thus needed, and where to derive requirements from. Further research topics were identified, including pre- production and post-production aspects, search engines for lifecycle data of things, long-term availability and exploitability of data, data ownership, safety, security and privacy issues, PSS interoperability across sectors, digital twin use cases. Also the "Factories of the Future 2020 Roadmap", created by the "European Factories of the Future Research Association (EFFRA)" names a number of related research issues on PSS interoperability within its chapters on

collaborative product service systems design environments for SME involvement, product service simulation for sustainability impact, data collection, analysis and anonymization during product usage. 


\section{Service-Oriented Architectures for Smart Manufacturing}

\author{
Nenad Ivezic \\ National Institute of Standards and Technology \\ Gaithersburg, MD, USA
}

\author{
Boonserm (Serm) Kulvatunyou \\ National Institute of Standards and Technology \\ Gaithersburg, MD, USA
}

Today, manufacturing enterprises are supported by large, monolithic systems assembled in a hierarchical fashion, following rigid enterprise architectures. However, as both (1) the components, equipment, and devices gain in networking, processing, and intelligence capabilities and (2) the service-oriented architecture-based methods gain in systems management capabilities, the capabilities of the monolithic systems and even devices can now be offered as services of varying complexity. The SOM vision is that the industrial applications will be rapidly and costefficiently composed from the new capabilities offered as message-exchanging services in the cloud. Transitioning to the new SOM world, however, presents many challenges.

Among the challenges, a leading one is achieving interoperability of services in the SOM systems. For that reason, we focus on message standards - a key part of systems integration solutions where interoperability of message-exchanging services is affected. The key issue is that the message standards are currently hard to use. Two causes for that are (1) message standards complexity and (2) lack of support for their reuse. Today, each case of message standards use for systems integration is a one-of situation, with no ability to reuse experience and information from similar integration cases.

To address this issue, we need to re-engineer the message standards development and usage approaches. We are following a model-driven approach, with the benefits at both design and implementation phases of the messaging standard use - to create message profiles for specific integration requirements. First, at the time of gathering systems integration requirements, a business process analyst can gather integration requirements as digital, computer-processable specification - we call it business process context. This activity is supported by formal models of business processes, repository of these business processes, and tools that allow analyses of these processes.
Next, at the time of actually creating a message profile based on the integration requirements, a computer-assisted process called 'semantic restriction' is where an integration engineer applies the business process context information to syntax-independent standard message definition to get the actual profile - a syntax-independent, context-specific profile message. This is possible by relying on syntax-independent standard message definitions that are accessible from a modelbased standards repository. As a result, it is a matter of automated process to generate a language specific representation of the standard profile, for a given target language specification.

Such a re-engineered process offers potential for managing complexity of message standards by reuse of the knowledge and experiences across the integration efforts and standards usages. At the time of integration requirements gathering, there is opportunity to collect and share, in digital form, the business process models and contexts derived from these models that inform message profiling. In addition, at the time of semantic restriction, there is opportunity to collect and share the decisions that go into syntax-independent, contextspecific profile messages for future reuse in similar integration cases. This ultimately will improve the quality of the message standard and accelerate the integration processes.

At NIST, we are developing tools to support this model-driven approach to development and use of messaging standards. The Semantic Refinement Tool (SRT) is focused on semantic refinement activity and standards representation generation. The Business Process Cataloging and Classification System (BPCCS) is focused on supporting the SRT tool by managing business process models and their constraints to provide basis for reuse in the form of business process contexts. This presentation will review the motivation, approach, and functionalities of the two tools. 


\section{Digital Twins for Through-Life Support - Setting the Requirements}

\author{
Dr. John Erkoyuncu \\ Through-life Engineering Services Centre, \\ Cranfield University, Bedford, Beds, UK
}

\author{
Prof. Rajkumar Roy \\ Through-life Engineering Services Centre, \\ Cranfield University, Bedford, Beds, UK
}

The development of complex computer modelling, simulations and connectivity between products introduces new and unique opportunities to elicit service value. The opportunities, risks and requirements of such technology are not fully understood. Accordingly, this presentation will setout a roadmap for the use of digital twins within a through-life perspective while focusing on the inservice phase of complex engineering products. Digital Twin (DT) is defined as a digital representation of a physical item or assembly. The digital representation holds data from multiple sources across the product lifecycle. This data can be analyzed to predict current, future state and simulate conditions in both design and operational environments.

The 'Global Horizons Report' (United States Air Force Global Science and Technology Vision, 2013) states that the DT could reduce development cycle time by $25 \%$. However, yet there is no demonstrable success for DTs as it is still a notional concept held back. However, as yet there is no demonstrable success for DTs as it is still a notional concept held back. There are a number of fundamental research gaps that cause this e.g.:

- Developing an unbroken data link (e.g. between the engineering $\mathrm{CAD}$ model loaded in the $\mathrm{CNC}$ ).

- A lack of models available that elaborate from a real system, condition or environment used to generate results that can simulate this same condition or environment.

The talk will focus on, if we had a through life 'digital twin' or virtual avatar of a product design and/or an individual physical product, what...
- $\quad$...would its uses be?

- ...benefits would it bring?

- ...else would be required to achieve those benefits?

- ...characteristics and capabilities would the digital twin need to have?

- ...impact would the current stage in the product life cycle have on the above?

The talk will focus on two digital twin demonstrations in areas of remote maintenance and design for service. The demonstrators will allow to:

- Define the cardinal decision points, people and questions that need to be addressed.

- How to design a 'digital twin' that would support more effective decision making.

- Verify the value of the 'digital twin' with selected user communities.

- Map the data sources, transportation and processing routes required to support the 'digital twin'.

The presentation will also highlight some of the key areas that DTs can help to improve, including:

- Tailored maintenance delivered specific to a component serial number.

- Better informed decision making capability in the design stage to future-proof the implications of proactive maintenance actions.

- Just in Time (JIT) spares provisioning from the supplier reducing customer requirements to warehouse and administer spares, and reduced maintenance burden on spares with a 'shelf life'. 


\title{
Supply Chain MBE/TDP Improvement
}

\author{
Andrew Hall \\ Rolls-Royce North America \\ Indianapolis, IN, USA
}

The development of model-based enterprise (MBE) methodology, the use of the model-based definition (MBD) as the conduit for product data communication and dissemination, and the concept of the Digital Thread of contiguous product data through the product lifecycle have gained increasing awareness within U.S. Government acquisition and procurement communities and the U.S. manufacturing sectors. Model-based methodologies can offer significant improvements in communication among the participants in product lifecycle workflows and improvements in accuracy and efficiency in moving and using product data at many different enterprise levels. Yet, it requires both technological and methodological advancement to realize the true benefits, and changes in how organizations work to make those benefits last.

An organization becomes a MBE organization when they effectively utilize model-based definitions to drive product lifecycle processes, and to more effectively make business decisions - not only design, but also supplier interactions, sustainment and maintenance, or technical publications.

MBE can offer significant long-term time and cost savings by enabling enriched product definitions, enterprise wide information integration, and data re-use through supply chain and the entire product lifecycle. It can also facilitate a systematic framework for knowledge sharing through the internal and external supply chain.

Because of the complexity of implementing MBE through the supply chain, it is crucial to establish standards for both processes and data formats among different PLM/CAD tools and model-based work practices among companies. The primary objective of this project will not focus on developing completely new technologies. Instead this project aims to utilize current and emerging MBE technologies to achieve substantial change by stretching product definition methodology beyond part geometry and into annotated semantic (i.e., associative) models, behavioral parameters, and contextual definition throughout the product lifecycle. The proposed program objectives are:

- Improve knowledge/data sharing through supply chain

- Accelerate maturation of full MBD technology via AP242

- Accelerate maturation of DMC infrastructure

- Improve MBD training curriculum at colleges and industry

- Collaboration with America Makes to apply MBD for additive manufacturing processes

This is achieved by addressing two main activities:

- The PDM/PLM/MBE interoperability area, the process from the part modeling, PLM, all the way to actual model consumption in the manufacturing stage will be developed and demonstrated through a simulated production environment.

- The bi-directional TDP data flow process management and reuse of information through the supply chain, including using the mobile platform. Systematic knowledge capture and sharing through the supply chain will be developed and demonstrated.

The team consists of four levels of suppliers- Airframe OEM (Lockheed Martin), engine OEM (Rolls Royce, program lead), Internal/External Inspection (RollsRoyce inspection group and Zeiss), SME for additive manufacturing (3rd Dimension). PLM/CAD/CAM technology providers include ITI Transcendata, Anark, Siemens and Microsoft. Academia member is Purdue University. NIST has offered to be the technical consultant; NCDMM and Y-12 from the Oakridge Lab will also be an observing member of the team to advice on additive manufacturing and cybersecurity issues respectively. 


\section{System Analysis Integration for Smart Manufacturing and Logistics Systems}

\author{
Conrad Bock \\ National Institute of Standards and Technology \\ Gaithersburg, MD, USA
}

\author{
Timothy Sprock \\ National Institute of Standards and Technology \\ Gaithersburg, MD, USA
}

Designing and operating modern manufacturing systems is difficult because information about the systems and their analysis is expressed in redundant and incompatible ways across multiple engineering disciplines (such as electrical, materials, and process models). The Systems Analysis Integration for Smart Manufacturing Operations Project supports smarter manufacturing by enabling faster and cheaper integration of manufacturing systems models and engineering analysis models. The project will deliver methods and protocols for unifying discipline-specific engineering analysis information and integrating that information with existing unified systems modeling information. Some of the areas currently being addressed are physical interaction and signal flow simulation, finite element analysis, and discrete event simulation and optimization for manufacturing and logistics.

As product complexity increases and lifecycles shorten, design of manufacturing and logistics systems early in the product lifecycle are critical to success. Manufacturing and logistics engineers must create and analyze system designs in a cost-effective manner throughout the product lifecycle. Industrial engineers have a broad variety of analysis methodologies to support design and decision making for manufacturing and logistics systems. Most of these require significant time and expertise to manually construct analysis models, such as simulation models. These methodologies would be simpler and less time-consuming with automatic formulation and construction of analysis models from an independent representation of the system. Multiple analysis models could be generated from a single system model, rather than constructing a single analysis model by hand, as is commonly done. This requires manufacturing and logistics systems to be modeled at the same level of detail as the product being delivered.

Through formal system-analysis integration methods, analysis models that conform to a standard definition of the system can be targeted for reuse and automation. Most analysis models commonly used in manufacturing and logistics are constructed from some abstraction of the system. Reusable abstractions can be captured in system reference models and analysis libraries. Our research seeks to harvest the underlying abstractions, make them formal, and provide a method to automate the formulation of analysis models from the abstraction. Reusability of system-analysis integration methods relies heavily on constructing the right abstraction of the domain. If the abstraction does not apply to a broad enough class of systems, then the return on the investment to construct the transformation methods and tools is not substantially greater than integrating each class of systems individually.

We have developed a multi-layer abstraction that can be reused across a large family of related systems called Discrete Event Logistics Systems (DELS), which include manufacturing and logistics systems and other auxiliary systems necessary to enable modern enterprises. They are dynamic systems that transform discrete flows through a network of interconnected subsystems. These include systems such as supply chains, manufacturing systems, transportation networks, warehouses, and health care delivery systems. Traditionally, each specialized kind of DELS has been regarded as a distinct class of systems requiring its own dedicated research and development. However, these systems share a common abstraction, i.e. products flowing through processes being executed by resources configured in a facility (PPRF), and they appear together in integrated models of the enterprise. For example, production systems might integrate storage and fulfillment capabilities as well as material handling and transportation systems, and supply chains might integrate flows between warehouses, transportation systems, and manufacturing or health care facilities. 


\section{MBE, MBSE and MaaS - Unleash Your Supply Chain}

\author{
Dr. J Simmons, PhD \\ Phoenix Integration \\ Washington, DC, USA
}

\author{
Anthony Davenport \\ Phoenix Integration \\ Baltimore, MD, USA
}

Utilizing the combination of MBSE and MBE, Systems Engineers are now able to utilize tools like ModelCenter to connect their domain expert's high fidelity simulation models (both physics and costs) directly to their systems engineering models - creating an environment for making decisions at all levels of the design. The next big step is to look toward Model as a Service (MaaS), where OEM simulation workflows are connected directly to supplier-based manufacturing simulation workflows to drive down production costs while still hitting performance goals; all while ensuring Intellectual Property is appropriately managed at all levels. Phoenix Integration has 22 years working with major defense OEMs helping them realize the benefits of MBE-MBSE-MaaS.

Over the last decade or more, there has been a steady increase in the use of modeling and simulation technologies for engineering analysis and design. These technologies have allowed engineers in a wide range of industries to shorten the design cycle and reduce product development costs by minimizing the use of physical prototypes. The full benefits of modeling and simulation, however, have not yet been fully realized.

To achieve the full benefits of modeling and simulation, organizations must more thoroughly adopt Model-Based Engineering (MBE) design processes. In a Model-Based Engineering environment, simulation is used early and throughout the design process to support critical design decisions and tradeoffs. This allows design problems to be avoided before they occur and avoids costly and time consuming rework. By studying multiple alternatives early and often, intelligent decisions can be made and optimal solutions can be found.
When bi-directionally coupled with the system engineering subset MBSE (Model Based System Engineering), MBE offers the ability for systems engineers and domain experts to realize changes throughout the entire product lifecycle to include conceptual design, performance analysis and manufacturing analysis.

Phoenix Integration, a 20+ year veteran in MBE, has developed an innovative software framework called ModelCenter for flexibly meeting the needs of Model Based Engineering. Working within the ModelCenter framework, engineers can create and maintain a library of modeling and simulation tools and simulation workflows, automatically execute the workflows, leverage high performance computing resources to perform trade studies and ask "what-if" questions, and archive, manage, and share the resulting data and metadata across their organization and their supply chain.

Engineering organizations using ModelCenter can achieve large Cost and Productivity improvements by:

Reducing Errors caused by ad-hoc and manual processes

Improving the Efficiency of their engineering analysis and design processes

Identifying Potential Problems Early when they can be corrected without large costs

Making Better Design Decisions

Creating Better Products

Capturing Engineering Knowledge

Enabling Collaboration among team members to include Supply Chain

Reusing Data and Knowledge on future projects 


\section{Functional Verification of Complex Engineering Designs using System-Level Modeling}

\author{
Paul Goossens \\ Maplesoft \\ Waterloo, Ontario, Canada
}

\author{
Joydeep Banerjee, PhD \\ Maplesoft \\ Waterloo, Ontario, Canada
}

\author{
Andy Ko, PhD \\ Phoenix Integration \\ Blacksburg, VA, USA
}

As engineered products become increasingly complex, manufacturers are forced to rethink their design processes, and there has been a growing adoption of the systems design $\&$ develop process. This approach provides a framework whereby complex multi-disciplinary systems can be broken down into subsystems to a level of granularity that allows various design teams to focus on the smaller subsystems, then carefully integrate them and test their behaviors against specifications throughout the process.

In order to reduce the cost and disruption caused by late-stage design changes, there is a lot of attention is being paid to processes and tools to help identify and address possible design issues as early in the process as possible. In particular, we have the emergence of Model-based Systems Engineering (MBSE) tools to manage the vast amount of interrelated design requirements, and Model-based Design (MBD) tools to allow engineers to develop conceptual designs of the system to address those requirements.
MBSE promises to manage the design requirements of complex systems in a tightly integrated fashion, allowing for efficient requirements validation throughout the design process. A detailed casestudy will demonstrate how a change in the specified operating conditions for an electric vehicle can be simulated to reveal the impact on the battery design requirements very early in the design process. The ModelCenter integration environment allows the combination of design requirements in SysML with multi-domain system models in MapleSim for functional verification of the design concept. This highly integrated environment enables the engineering design team to readily verify a proposed system against its specification long before investing in the prototyping stage.

The ability to automate the verification of functional requirements is a major step forward in the system-design process, significantly reducing project risks and unexpected late-stage design changes.

More information about this case-study can be found at: $\underline{w w w . m a p l e s o f t . c o m} \backslash \mathrm{mbse}$ 
Systems Engineering

\title{
Developing a Model Based Enterprise (MBE) Strategy within Army Organizations
}

\author{
Gregory A. Harris, Ph.D., P.E. \\ Auburn University \\ Auburn, AL USA
}

The world of product and process data is changing at a rate faster than most large organizations can keep up. New initiatives such as the Digital Manufacturing and Design Innovation Institute (DMDII) are developing solutions to many of the gaps and issues that have hindered the manufacturing industry from accessing the power of the completed the Digital Thread. The Army's industry partners have managed to stay abreast of the movement toward a digital manufacturing environment, but the Department of Defense (DoD) and component branches and agencies have not able to maintain the level of investment necessary to develop the same understanding and capability of their industry partners. This can be attributed to the reality that the need to work with models has not reached the level of urgency that other issues in the development and fielding of DoD systems achieve in the day-to-day activities of these programs. However, there is a genuine issue that the inability to perform required functions using models is going to cause in the very near future.

Some DoD industrial partners have indicated that they no longer intend to provide 2-dimensional (2D) drawings as a deliverable on contracts but instead will be delivering 3-dimensional (3D) CAD models. Most Army organizations currently do not possess the capability to receive and utilize $3 \mathrm{D}$ models to perform the functions for which they are responsible. To achieve the level of proficiency necessary to support the warfighter, a strategy for the use of the MBE tools and a methodology is needed. In 2010, the JDMTP AME Subpanel described MBE as "building the digital thread" in the
ManTech Strategic Plan. It states that the MBE challenge is to "drive a continuous flow of integrated design, analysis, and manufacturing information through the product/system life cycle." The subpanel refers to MBE as including advanced modeling and simulation, design optimization tools, virtual prototyping, and data standards efforts. If MBE is an integrated and collaborative environment, founded on 3D product definition (Model Based Definition - MBD) shared across the enterprise, enabling rapid, seamless, and affordable deployment of products from product concept to disposal, it is important that Army harness the power of MBE to execute required function in the support of Army systems.

Key factors required to implement MBE across Army organizations include a functioning enterprise Product Data Management (ePDM) system, documented business processes to guide MBE tool selection and configuration, policy regarding the acquisition and use of 3D MBD, consistent leadership emphasis to affect cultural change, and digital product data (including fully annotated 3D models). MBE tools and processes must be common, but can be tailored to each organization and site based on mission, but, an Enterprise approach is critical. This will not be achieved through chance and random application; it will take a strategic plan to guide and manage the initiative and subsequent culture change. Additionally, there must be a resource for Army organizations to call upon to assist in the implementation. This presentation will introduce the concept of the Model Based Enterprise Capability Center. 
Systems Engineering

\title{
The Model Based Enterprise \& Systems Engineering: A Black Swan Inspired Perspective
}

\author{
Lisa D. Murphy, Ph.D. \\ Siemens PLM \\ Atlanta, GA, USA
}

In his groundbreaking book The Black Swan, Nassim Taleb introduces the concept of "silent evidence" while discussing cognitive barriers to understanding highly improbably events. The premise of silent evidence is that we extrapolate not from the starting base to today but from today's outcome backward, leaving us with a misleading understanding of the likelihood of relatively ordinary events, as we only see today what was successful, not all the dead-ends, abandoned paths, and solutions not pursued.

By starting to define what information supports the Model Based Enterprise (MBE) from the product definition needed for manufacturing, we may experience the silent evidence fallacy as we move upstream into the product development flow. Factors that make MBE silent evidence significant include the nature of the product development process that moves from abstract to concrete, that the level of uncertainty increases as you go backward into the development history, that legacy systems often have different paths, and may have different information, different tools, and different data formats. Notably, even new designs may have elements starting at different times and maturing at different rates. And our methodologies for product development are seldom pure as the process diagrams suggest, but end up with a mix of approaches that often remain incompletely documented.

The number and types of models produced is often difficult for people not involved in managing the data across the lifecycle to see, and the relationships between them are not always as direct and linear as we imagine (a side effect of silent evidence). Models that used to be expensive and time consuming to produce are now produced by the thousands rather than the dozens. Model of a particular type are no longer only found in a given development phase; for example, we are seeing the same type of 1-D physics model being used in the systems engineering and architecting phase to identify the variables that matter for optimization so they can be factored into requirements and passed along to design and testing activities. This is the equivalent of virtual integration, which is like starting up the right side of the Systems Engineering V model from the Systems Architecting step, rather than from Implementation.

It has been a promise of MBE (and a goal of engineering and program management) to replace costly physical tests with models and simulations, and in many cases, that effort is progressing. For example, the large, cumbersome, time consuming, and expensive test called the Iron Bird where physical instances of all controls are run the same distance with the same turns and angles they would have upon installation has now been replaced by one aircraft manufacturer with a Virtual Iron Bird simulation model.

Another experience of modeling in MBE related to testing is less familiar, and that is modeling to simulate the physical test rig with the test article in it. While someday it may be possible to do away with all testing, until then, tests that are costly and dangerous such as fuel cells can have critical cases simulated by modeling the fuel cells in the test rig, allowing tests to be run faster and safer. These models are significant in the development process but are very unlikely to appear in a product-based view of a product's models.

The Black Swan concept of silent evidence can help remind us that the digital thread looks different moving forward than it does moving backward. As the nature, types, and uses of models continues to expand across the system development life cycle, we will have to take care to avoid a digital tangle. 


\title{
STEP for Downstream Consumption
}

\author{
Robert Kirkwood \\ President, Integration-Guard \\ Nashua, NH, USA
}

\begin{abstract}
1. Overview
The speaker shows a new technique: Virtual Persistent Identifiers (VPI) to enable sustained integration (associativity) using STEP files as they are currently exported by any major $\mathrm{CAD}$ vendor. The technique is shown to work for Casting Design, NC Programs, FEA-Solids, and FEA-Shells. In each case the files are exchanged using STEP files as exported/imported to a variety of CAD applications. Sustained integration meaning that after the initial integration, successive iterations only require an incremental effort.
\end{abstract}

\section{Problem statement}

MBE implementations face a dichotomy regarding CAD formats. Standard format (e.g. STEP) versus proprietary formats. Proprietary formats have one remaining advantage, they are better at staying integrated over successive design iterations of the given format. The sooner that advantage has been neutralized, the sooner we may expect accelerated adoption of STEP for MBE.

\section{Conclusions}

1. STEP data as currently exported by every CAD vendor is already sufficiently to support sustained integration (associativity).

2. The VPI technique is a new technique to enable sustained integration. It mitigates most of the rework trauma associated with design changes via export/import of neutral formats.

3. Broken integration (associativity) can be repaired

4. The ' $E$ ' in MBE does not mean that a daunting enterprise-wide implementation is the only option.

\section{Recommendations}

1. Manufacturers: Permit vendor compliance with company MBE standards via
STEP. Keep the same functional standards, just recognize that STEP might satisfy those standards.

2. CAD vendors: Allow that data might be imported with valid persistent identifiers.

3. CAD vendors: Use the optional attribute for entity names to export persistent identifiers.

4. All: Educate your executives The issue is IDs/namespaces, not translation/format

5. All: STEP test parts could include some cases of successive versions.

6. All: Send challenge cases

\section{Supporting information}

Proprietary integration and point-to-point translators rely on absolute persistent identifiers on the various geometric entities. This persistence enables downstream applications referencing the model data to stay integrated across successive versions. Each translation to/from neutral formats like STEP re-sequences those identifiers with each iteration, rendering the identifiers different from those in the CAD system and no longer persistent. Virtual persistent identifiers to not attempt to use the same identifiers as are in the source CAD model. Instead, the identifiers in each successive version are mapped back to the originally translated identifiers. Thus, the virtual persistent identifiers present themselves to the downstream application as being persistent even though they differ from those in the originating CAD system.

No engineer wants to be burdened with a new task managing identifiers in his imported geometry. Design Change Vectors $\left(\mathrm{DCV}^{\circledR}\right)$ provides notation and tools allow an engineer to assure virtual persistent identifiers without significant new effort. Design Change Vectors allows changes to solid models to be recognized (subtraction), added (merging), and created as parallel to other changes. 


\title{
Best Practices for Creating MIL-STD-31000A Technical Data Packages (TDP) using (3D) PDF and STEP Executive Summary
}

\author{
Jerry McFeeters \\ 3D PDF Consortium \\ Cincinnati, OH, USA
}

In early 2016, the 3D PDF Consortium began planning test round two for the 3D PDF Implementer Forum. Early in the planning stages it became apparent that there was a need to extend beyond the interoperability testing used by traditional implementer forums. The Consortium members wanted to extend the testing to include the document capabilities of the PDF format. A quick survey of our membership determined that the test round should be focused on testing the ability to create an optimized TDP conforming to MIL-STD-31000A using the STEP and PDF formats.

In order to prepare for this unique undertaking, the 3D PDF Consortium solicited and received from PDES, Inc. members, MBD360, Action Engineering and others who were intimately familiar with MIL- STD-31000A. After several meetings, the project scope was defined and the Implementer Forum was initiated in 2016.

One of the products of this effort is a paper containing Best Practices for utilizing STEP and 3D PDF to meet MIL-STD-31000A standards. The following is an outline of some of the best practices that are recommended:

- Use Adobe Acrobat DC or Adobe Acrobat Reader DC

- Use the Best Standards for 3D

- $\quad$ ISO 32000 (PDF)
- $\quad$ ISO 14739 (PRC)

- ECMA-363, 3rd Edition (U3D)

- Add Structure Using Portfolios and Attachments

- Make sure all attachments have a description

- Use folders to logically organize attachments

- Use hyperlinks to easily open attachments from inside the portfolio

- Attach CAD and/or STEP file

- While PRC can represent precise geometry, very few programs can read this data from a PDF file

- Workflows that require a precise CAD model, such as CAM, should attach a neutral STEP file and/or native CAD file

- Use JavaScript to support ANSI Y14.41

- Ensure Your Document Is Portable

In conclusion, it was determined that 3D PDF has unique features that make it an excellent format for TDP including Collections/Attachments, XFDF support, and JavaScript support. Recommendations for future rounds of the 3D PDF Implementer Forum includes the exploration of ways to more tightly integrate $3 \mathrm{D}$ PDF with STEP and other open formats. 
Design

\section{Design Decision Support for MBE with Information Modeling}

\author{
Douglas Eddy \\ University of Massachusetts at Amherst \\ Amherst, MA, USA
}

\author{
Sundar Krishnamurty \\ University of Massachusetts at Amherst \\ Amherst, MA, USA
}

\author{
Ian Grosse \\ University of Massachusetts at Amherst \\ Amherst, MA, USA
}

The advancements in additive manufacturing (AM) and the renaissance of interest in semantic representation for knowledge management in support of the product design digital thread advances have led to the convergence of several main thematic research scopes to support advanced manufacturing. Along these lines, ongoing research at the Center for e-Design at UMass Amherst has focused on parallel and interconnected research themes addressing advancements in the state-of-the-art in AM, focusing on the development of a product design architecture by inclusion of design for AM concepts in an information model at early design stage. The overall goal of our project is to model information associated with a product's digital thread based on the context and meaning of such information in a way that facilitates robust and reliable early design and process decisions. This is a compelling need to address currently due to the emerging trends in large amounts of data and information to manage, along with the paradigm shift in the design of products caused by the everexpanding capabilities of additive manufacturing (AM) processes. Thus, the foundation of this work is an ontological framework tool and approach that can both organize design and manufacturing knowledge in a reusable way that facilitates accurate early decision-making. Our work introduces concepts related to AM within the existing Manufacturing Service Description Language (MSDL) ontology, developed at Texas State to identify process plans for conventional manufacturing. Our approach utilizes both part and process information to identify which combination of processes can produce a desired product. Here, first order logic rules provide the fundamental basis in the selection of the bestsuited candidate process combination in product realization, thus enabling seamless comparison of AM with other manufacturing alternatives.
Given the aforementioned paradigm shift in early product design decisions caused by rapidly improving AM capabilities, early process selection is now significantly more consequential. Thus, better and different methods must decide early based on available information whether to design a part for AM or conventional manufacturing. To this end, our eDesign team at UMass Amherst has developed a formal decision-based design support system focusing on the unique differences between design for manufacturing and design for additive manufacturing. A salient feature of this approach is its unique ability to identify inflection points at various production quantities at which the design of choice can change from conventional manufacturing to AM and viceversa. To test applicability of our approach in a practical industry environment, we collaborate with FTL Labs to fill a specific need. Here, the ontological approach extends to relate CAD-based design features to metal Direct Energy Deposition AM process capabilities. The ability to reason upon such information can provide visual prompts to a designer in a CAD interfaced software tool about any needs for design revisions to enable fail proof manufacturing. Furthermore, a reusable knowledge base can expand over time to improve process predictability in advance by enhanced querying and reasoning.

Having developed the conceptual approaches to both manage the knowledge and utilize that knowledge in early stage decisions, a remaining challenge concerns developing the relationship of domain-specific information in a framework consistent with other domains. Towards this goal, our future work will focus on building and connecting established engineering design domain concepts within the Basic Formal Ontology to test for consistency and reasoning capability within this established high-level knowledge foundation. 


\title{
A Digital Product Realization Revolution Enabled by Persistent Model-Based Product Characteristics
}

\author{
Curtis W. Brown \\ Honeywell FM\&T* \\ Kansas City, MO, USA
}

\begin{abstract}
* Department of Energy's Kansas City National Security campus operated and managed by Honeywell Federal Manufacturing \& Technologies, LLC under contract number DE-NA0002839.
\end{abstract}

The purpose of this presentation is to advocate for those that are on the Model-Based Enterprise (MBE) journey to travel along a newer route with the most impactful benefits. The proposed MBE way must be via the Digital Product Acceptance Road by means of the "new" Model-Based Product Characteristics Bridge. This presentation reviews various technologies and digital interoperability standards that have arrived or matured that can revolutionize closed-loop automation within a MBE business strategy. Specifically, the presentation promotes the fundamental use of persistent product characteristics, digitally consumed early in the product lifecycle and throughout the enterprise. The results will transform manufacturing quality into an agile and valued contributor to the manufacturing business.

Manufacturing Quality is a customer requirement. It is not free, nor is it optional; however, it can be achieved faster, better, cheaper, and smarter with model-based innovations and standards-based digital interoperability. Successful manufacturing organizations recognize that production definition is a valuable asset for their companies and it is necessary to realize and accept their product. Traditionally, this was accomplished through 2D static drawings. Today, this product definition can be captured as a digital product definition (DPD). The DPD, aka model-based definition (MBD), uses a computer-aided design system that fully describes the shape geometry, metadata, and any product and manufacturing information (PMI) necessary to help communicate design intent.

An added advantage for transitioning to MBD is the significant opportunity to enable the Quality organization with Model-Based Product Characteristics. A Product Characteristic is a tolerance or specification applied to a feature or product that requires verification to assure that the product satisfies requirements. Product Characteristic designators and their assocated criticality levels are essential for quality and inspection processes. At present, this information is identified and labeled just-in-time at the inspection department thru the process of "ballooning the drawing". Imagine the case where this product, manufacturing $\&$ inspection information (PMII), (PMI with model-based product characteristics), could be defined digitally and earlier in the lifecycle within the MBD and persists throughout the enterprise.

A Bill of Characteristics (BoC) is a listing of product characteristics required for manufacturing quality to consume and verify product acceptance. A complete MBD with PMII can generate a BoC. Furthermore, the Quality Information Framework (QIF), an American national standard for digital interoperability, can effectively represent a modelbased BoC. This model-based BoC information would drive downstream processes and then allow for closed loop measurement results to be fed back to the MBD. 


\title{
Measurement Data Analytics using QIF
}

\author{
Daniel Campbell \\ Capvidia \\ Sugar Land, TX, USA
}

\begin{abstract}
Modern metrology systems consist of a patchwork of various individual hardware and software packages, each of which produce massive amounts of Data. Globalization of industry and vast, connected supplier networks help to add to the available amount of Data. With the advent of Industry 4.0, Data exchange becomes a key part of industrial processes.
\end{abstract}

A significant problem we now face is: how does an organization best leverage all this available Data? Under current practices, most measurement Data is not exploited at all, and when it is, it is typically accomplished via limited, proprietary tools or complex PLM customizations. To extract Knowledge about industrial processes from this vast amount of measurement Data, it must first be organized into Information with meaning and context.
There exists a better solution which enables Model Based Enterprise to fully leverage all its measurement Data. The context and meaning behind measurement Data can be provided by the Quality Information Framework (QIF). QIF is an XML-based ontology for manufacturing Data, all built on semantic links to the 3D model. This solution arose organically via a body of industry experts ranging from manufacturers (end users), software vendors, research organizations, and National Measurement Institutes, all coordinated by the Dimensional Metrology Standards Consortium (DMSC).

We showed how software tools can be used to analyze enormous repositories of measurement Information in the QIF format. Advanced analytics and Business Intelligence (BI) methodologies can be applied towards this Information to understand trends, root causes, and otherwise increase manufacturing Knowledge. 


\title{
Applying visual variables to manufacturing data for enhanced decision-making
}

\author{
William Z. Bernstein \\ National Institute of Standards \& Technology \\ Gaithersburg, Maryland, USA
}

Since Jacques Bertin's seminal work on visual variables [1], significant effort has been focused on mapping visual primitives, such as color, position, and size, to domain-specific data. To realize an appropriate mapping of visual variables, several development procedures are required, including initial design, prototyping and implementation, interface evaluation with human subjects, as well as integration into existing computer systems. These steps are no different when applying the same set of visualization principles to manufacturing. However, manufacturing presents unique challenges for interface developers, such as the connection of disparate databases, complexity in different stakeholder perspectives and roles, domain-specific data representations, such as STEP and MTConnect, as well as the prevalence of significant data uncertainty.

In this presentation, we present three different efforts in applying guiding information visualization principles to make sense of manufacturing-related data. The three prototypes include an interactive exploration tool that allows users to make sense of manufacturing sensor data (i.e. MTConnect) and its relationships to design models [2], an interactive interface that allows users to explore relationships of key performance indicators and their underlying metrics [3], and a workflow for visualizing the similarities of capabilities of manufacturing processes [4]. All three instances use data elements and representations that follow existing standards, such as ISO 22400 and MTConnect. From these different visualization prototypes, we have begun to distill best practices for mapping visual variables to manufacturing data to enhance practical and direct decision-making. An initial set of these "design patterns" focuses on the development of visual analytics tools to aid in sustainable product design [5].

In the near-term future, we plan to install the Data \& Information Visualization \& Exploration (DIVE) Lab, to more deeply explore aspects related to human interaction and the evaluation of these interfaces. One of the main goals of the DIVE lab is to demonstrate our visualization prototypes and to test their utility on a distributed, collaborative system, where multiple stakeholders are interacting and exploring a common dataset from different perspectives based on their specific role. A practical use case here would be a machine operator on the shop floor detecting a problem at a workstation and then passing a message to a manager that can interact with metadata regarding that issue. We plan to implement a testbed version of this use case to explore new opportunities in developing interface design guidelines for such distributed collaborative environments. Our hope is that these best practices, or "design patterns", will quicken the design, prototyping and implementation processes of new visualization-based interfaces for data-driven decisionmaking.

\section{ACKNOWLEDGEMENTS}

The author thanks Kevin Li, Dr. Moneer Helu, Thomas Hedberg Jr., Allison Barnard Feeney, Dr. Michael Brundage, KC Morris, and John Horst, who all contributed to examples presented in this presentation.

\section{REFERENCES}

1. Bertin, J., 1983. Semiology of graphics: diagrams, networks, maps.

2. Bernstein, W. Z., Hedberg Jr, T. D., Helu, M., Barnard Feeney, A., "Contextualizing manufacturing data for lifecycle decision making." International Journal of Product Lifecycle Management. Under review.

3. Brundage, M. P., Bernstein, W. Z., Morris, KC, Horst, J. A., "Using graph-based visualizations to explore key performance indictor relationships for manufacturing production systems." Proceedings of the CIRP 2017 LCE. To appear.

4. Li, K., Bernstein, W. Z., 2017. "Developing a capability-based similarity metric for manufacturing processes." Proceedings of the ASME 2017 MSEC. To appear.

5. Ramanujan, D., Bernstein, W. Z., Ramani, K., 2017. "Design patterns for visualization-based tools in sustainable product design." Proceedings of the ASME 2017 IDETC/CIE. To appear. 


\title{
Model-based Visualization for Resistance Spot Welded Assembly Design
}

\author{
Kyoung-Yun “Joseph" Kim, Ph.D., Md. Tarique Hasan Khan, \\ Fahim Ahmed, and Gary Witus, Ph.D. \\ NSF Center for e-Design \\ Wayne State University
}

Resistance Spot Welding (RSW) is one of the most common sheet metal welding processes in various manufacturing industries including the automotive industry. However, the utilization of the RSW process data is still limited due to disconnected welded assembly design models and associated process data challenges. Model-based engineering provides an opportunity to combine and visualize the data from different lifecycle stages of a welded assembly product in a unified platform.

This study presents a model-based visualization approach to integrate RSW weldability knowledge with assembly models. For this study, a semantic weldability prediction method is used to effectively predict the weldability of RSW processes, while reducing the data inconsistency effects.

For this purpose, real industry RSW quality datasets are utilized to extract weldability decision rules with data mining algorithms. An RSW ontology, which serves as a formal assembly design model, is developed and builds a shareable RSW weldability knowledge.

A CAD system stores the geometric assembly design information and the STM (SpatioTemporal Mereotopology) ontology is connected to the design database in this work. Afterward, welded assembly design and weldability knowledge sets are extracted from the two ontology-based models and integrated with a X3DOM data to visualize the assembly design and weldability knowledge. To confirm if the unified platform can visualize the 3D assembly models with the predicted weldability, different welded assembly models are tested.

In the demonstration, the visualization platform successfully distinguishes assembly effects before and after welding and provides a formal and shareable assembly design knowledge including weldability. 


\section{A framework for an intelligent knowledge-based manufacturing diagnosis system}

\author{
Michael P. Brundage \\ National Institute of Standards and Technology \\ Gaithersburg, MD, USA \\ KC Morris \\ National Institute of Standards and Technology \\ Gaithersburg, MD, USA
}

\author{
Boonserm Kulvantunyou \\ National Institute of Standards and Technology \\ Gaithersburg, MD, USA \\ Thurston Sexton \\ National Institute of Standards and Technology \\ Gaithersburg, MD, USA
}

Various techniques are used to diagnose problems throughout all levels of the organization within the manufacturing industry. Often, the diagnosis is adhoc with no standard representation for artifacts or terminology (i.e., no standard representation for terms used in techniques such as fishbone diagrams, 5 why's, etc.). Once a problem is diagnosed and alleviated, the results are typically discarded or stored locally as paper or digital text documents. When the same or similar problem reoccurs with different employees or in a different factory, the whole process has to be repeated without taking advantage of knowledge gained from previous problem(s) and corresponding solution(s). In addition, when recording the diagnosis information, employees may miscommunicate over terms used leading to wasted time and errors. This research presents a framework for a knowledge-based manufacturing diagnosis system that aims to enable intelligent reuse and alleviate the miscommunication in the diagnosis information. The framework leverages diagnosis methods used in manufacturing and in the medical community, knowledge management methods, machine learning, and semantic technologies to enable such capability. A sequence diagram in Figure 1 illustrates an example of how a user may interact with the diagnosis system. In the figure, a user inputs effect(s) (e.g. symptoms) that are observed, which are compared against a thesaurus of previously used terms. Once these effect(s) are selected, potential causes (e.g. diseases) are retrieved from the diagnosis knowledge base with probabilities of occurrence. When a root cause is selected, the probability of the diagnosis in the knowledge base is updated according to a machine learning algorithm. The system presents and updates probabilities of a related corrective and preventative action associated with the diagnosis in the same way. In addition, not shown in the diagram is the ability to manage the thesaurus and knowledgebase with new or update terms, relationships between them, and diagnosis knowledge. This formalized intelligence maintenance framework will decrease time to perform maintenance activities as well as reduce errors in diagnosing production issues.

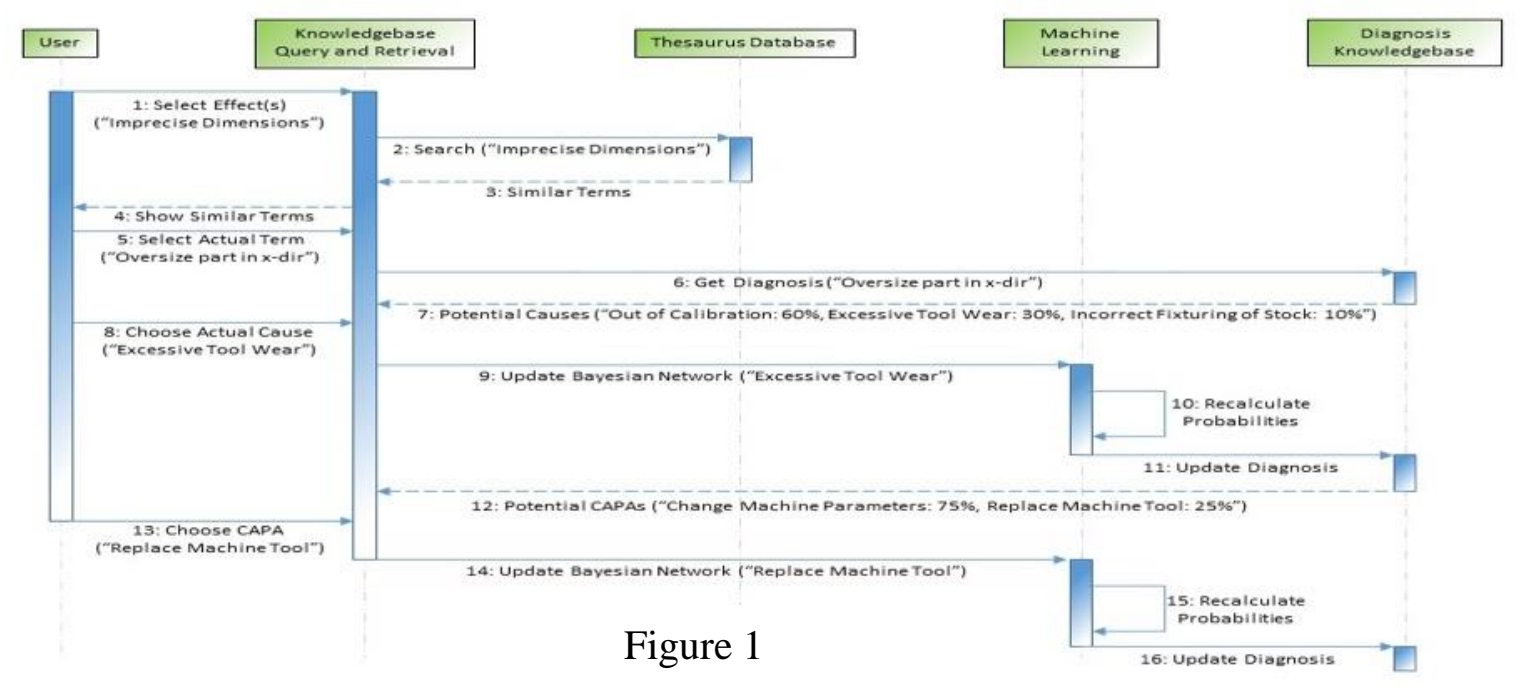




\title{
MBD Supplier Readiness
}

\author{
Jennifer Herron \\ Action Engineering \\ Lakewood, CO, USA
}

\begin{abstract}
Your organization may be "all in" - but what about your suppliers? Are your suppliers willing to keep pace or lagging behind? Are you keeping them "in the loop"? The benefits of Model-Based Definition (MBD) for your organization cannot be fully realized until your suppliers are operating at your capability level. Data from ROI of MBD surveys conducted by Action Engineering and Lifecycle Insights were shared. The high-level results from supplier survey data are:
\end{abstract}

- Manufacturing is a no-brainer because having models is better than only drawings when building parts

- Reduced time in quoting may yield significant savings
- How to accomplish digital inspection is still fuzzy. The following are needed: standard practices, software tools, training and product definition that supports digital inspection

- A properly instantiated Digital Enterprise may lead to production cost savings

The bottom line is: suppliers are ready to receive model-based data, but the organization is unaware of the readiness. Often an un-intentional wall is created between Engineering and willing suppliers that prevents the circulation of 3D digital data. Action Engineering's CEO discussed methods to open doors between engineering and suppliers. Many organizations discover that their source issue is the lack of resources within Procurement that can enable the flow of MBD data to willing suppliers. 


\section{Enabling Automated CNC Measurement on the Digital Thread}

\author{
William Sobel \\ System Insights \\ Emeryville, CA, USA
}

\author{
Larry Maggiano \\ Mitutoyo America \\ Aurora, IL, USA
}

\author{
Martin Hardwick \\ STEP Tools Inc \\ Troy, NY, USA
}

\author{
John Horst \\ National Institute of Standards and Technology \\ Gaithersburg, MD, USA
}

The digital thread will not be based on a single standard or single vendor solution. It will require many standards composed into a cohesive model of production across all dimensions including design, engineering, planning, scheduling, execution, measurement, and quality - to name a few. The challenge will be to harmonize the standards definitions and models to achieve an open end-to-end representation of the manufacturing digital thread.

Semantic standards provide the foundation of a composable software architecture where each standard provides capabilities that are necessary to the information flow. We will discuss good software architecture practices and how the separation of responsibilities between STEP, MTConnect and QIF allows for synergies by exploring an important use case for manufacturers. The use case is to perform accurate and timely dimensional measurements of part features, while those parts are being produced. Unsurprisingly, the multitude of information generated and consumed by metrology equipment and machine tool software is largely proprietary, and without expensive translation, changing software vendors and machine tool vendors can be very costly. The implementation of proprietary software- based on-machine dimensional metrology has reduced production costs for solution providers and manufacturers, despite the translation costs. However, widely adopted digital thread standards will reduce costs even further. Therefore, a joint working group between the
MTConnect Institute and the Dimensional Metrology Standards Consortium (DMSC) has been formed to provide a standards-based solution to this problem.

We describe how we are using the digital thread to enable the automated, hands free, and real time, validation of MBD design tolerances. The method uses STEP to describe design requirements, MTConnect to communicate machining results, and QIF to report inspection results and statistics. The method relates the three protocols by inserting Universally Unique Identifiers (UUID's) into the three types of files. When a point is touched, an enhanced MTConnect data stream communicates the UUID of the relevant tolerance with the sampled point coordinates. When sufficient points have been sampled, the CMM evaluates their coordinates and sends a report to the rest of the enterprise using QIF. The CNC and CMM can be at different locations, and both may be either actual or virtual. The measurements can be made using contact or non-contact systems, and they can take place on or off the CNC. The input to the process is a STEP AP242 file with tolerances on the part model. The output is a QIF file describing the results of evaluating the tolerances for a given part instance. The enabling technology is a digital twin server that monitors the results of the manufacturing processes and reports the coordinates of the sampled points in the design space of the part. 


\section{A Game Theoretic Approach to Minimizing Cybersecurity Risk}

\author{
Scott Musman \\ MITRE Corp \\ McLean, Va, USA
}

\author{
Andrew Turner \\ MITRE Corp \\ Bedford, Ma, USA
}

Approved for Public Release: Cases \# 16-3240 and 16-3460

Information and Communication Technology (ICT) systems are now ubiquitous in all aspects of our society. With an ability to create ICT incident effects via cyberspace, criminals can steal information or extort money, terrorists can disrupt society or cause loss of life, and the effectiveness of a military can be degraded. These threats have caused an imperative to maximize a system's cyber security and resilience. Protecting systems that rely on ICT from cyber-attacks or reducing the impacts that cyber incidents cause

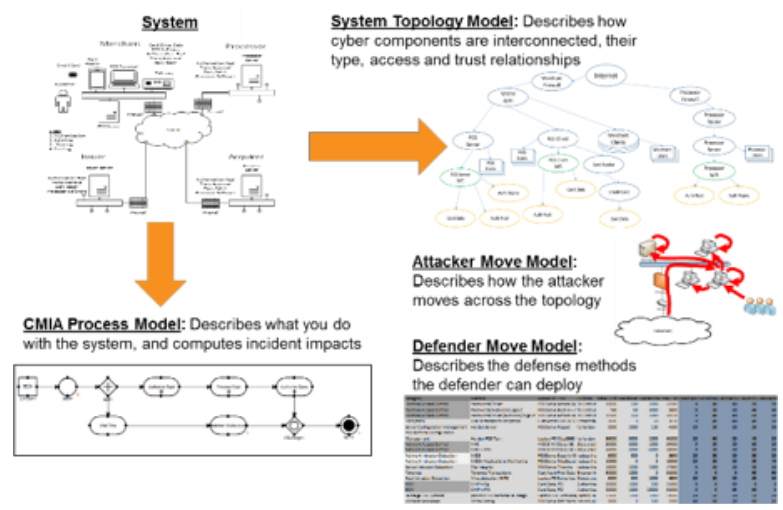

Figure 5: Models Used to Describe a System and Calculate its Cybersecurity Risks

(i.e. cyber security and resilience), is a topic of major importance. We describe an approach to minimizing cybersecurity risks which can be viewed as a form of model-based system security engineering. We describe a method and supporting software prototype that quantitatively identifies mission outcome focused cybersecurity risks and uses this metric to determine the optimal employment of security methods to use for any given investment level.
Our software maximizes a system's ability to operate in today's contested cyber environment by minimizing its mission risk.

The cybersecurity risk score we use is calculated by using a cyber mission impact assessment (CMIA) model to compute the consequences of cyber incidents, a system topology model, and a defender model (Figure 1).

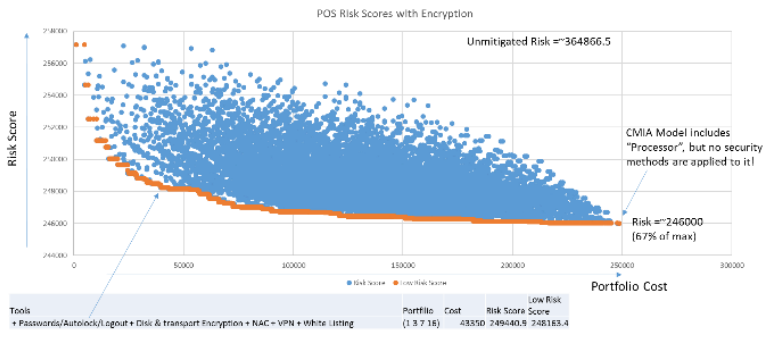

Figure 6: Mitigation Portfolio Analysis (showing Pareto Optimum Solutions)

When the system models are combined with an attacker threat model (a default threat model is provided) it is possible to estimate how likely attacks are to result in impacts. To do this the program takes into account the widespread interconnectedness of cyber systems, where defenders must defend against many multi-step attack paths and an attacker only needs one to succeed. We employ a game theoretic solution using a game formulation that identifies defense strategies to minimize the maximum cyber risk (MiniMax). employing the defense methods defined in the defender model. The program explores a game tree of attacker/defender options to determine how the attacker may circumvent any defenses employed by the defender. The result is a mitigation portfolio analysis (Figure 2) that determines the best employment of defenses for any investment level. This presentation describes the approach and the models used. 